HIGHLY ENANTIOSELECTIVE SYNTHESIS OF TETRAHYDRO- $\beta$-CARBOLINES AND TETRAHYDRO- $\gamma$-CARBOLINES VIA Pd-CATALYZED INTRAMOLECULAR ALLYLIC ALKYLATION.

Marco Bandini*, Alfonso Melloni, Fabio Piccinelli, Riccardo Sinisi, Simona Tommasi, Achille UmaniRonchi*

Dipartimento di Chimica “G. Ciamician”, via Selmi 2 - 40126, Bologna, Italy

marco.bandini@unibo.it; achille.umanironchi@unibo.it

\title{
General.
}

$\mathrm{CH}_{2} \mathrm{Cl}_{2}$, toluene, THF, $\mathrm{Et}_{2} \mathrm{O}$ and DMF were supplied by Fluka in Sureseal ${ }^{\circledR}$ bottles and used as received. All starting materials and reagents were obtained from Aldrich and Fluka and used without further purification. All the allylic carbonates were prepared following a procedure reported in the literature. ${ }^{1}{ }^{1} \mathrm{H}-\mathrm{NMR}$ spectra were recorded on Varian $200(200 \mathrm{MHz})$ or Varian $300(300$ $\mathrm{MHz}$ ) spectrometers. Chemical shifts are reported in ppm from TMS with the solvent resonance as the internal standard (deuterochloroform: $\delta 7.27 \mathrm{ppm}$ ). Data are reported as follows: chemical shift, multiplicity $(\mathrm{s}=$ singlet, $\mathrm{d}=$ doublet, $\mathrm{t}=$ triplet, $\mathrm{q}=$ quartet, $\mathrm{br}=$ broad, $\mathrm{m}=$ multiplet $)$, coupling constants (Hz). ${ }^{13} \mathrm{C}-\mathrm{NMR}$ spectra were recorded on a Varian $200(50 \mathrm{MHz})$ or Varian $300(75$ $\mathrm{MHz}$ ) spectrometers with complete proton decoupling. Chemical shifts are reported in ppm from TMS with the solvent as the internal standard (deuterochloroform: $\delta 77.0 \mathrm{ppm}$ ). GC-mass spectra were performed at an ionizing voltage of $70 \mathrm{eV}$. LC-electrospray ionization mass spectra were obtained with Agilent Technologies MSD1100 single-quadrupole mass spectrometer. Chromatographic purification was done with 70-230 mesh aluminiun oxide 90 mesh allumina or with 240-400 mesh silica gel. IR analysis were performed with a FT-IR spectrophotometer. IR spectra are expressed by wavenumber $\left(\mathrm{cm}^{-1}\right)$. Elemental analyses were carried out by using a EACE 1110 CHNOS analyzer. Analytical high performance liquid chromatograph (HPLC) was performed on a liquid chromatograph equipped with a variable wave-length UV detector (deuterium lamp 190$600 \mathrm{~nm})$, using a Daicel Chiralcel ${ }^{\mathrm{TM}}$ OD and AD column $(0.46 \mathrm{~cm}$ I.D. x $25 \mathrm{~cm})$ (Daicel Inc.). HPLC grade isopropanol and hexane were used as the eluting solvents. Optical rotations were determined in a $1 \mathrm{ml}$ cell with a path length of $10 \mathrm{~mm}\left(\mathrm{Na}_{\mathrm{D}}\right.$ line). LC-electrospray ionization mass spectra were obtained with Agilent Technologies MSD1100 single-quadrupole mass spectrometer. Melting points are uncorrected. 


\section{Preparation of benzyl-(1H-indol-2-yl-methyl)-amine}

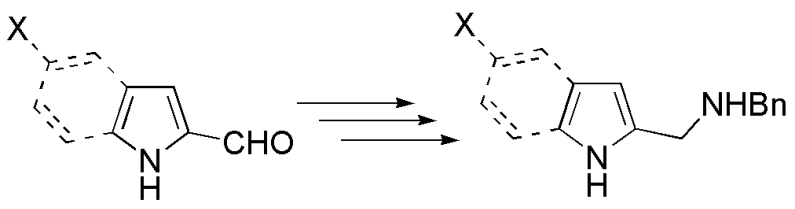

To a mixture of $4 / 5^{1}$ (5 mmol, 1.0 equiv), and $\mathrm{MgSO}_{4}$ (5.0 equiv) in toluene (30 mL), benzylamine ( 1.5 equiv) was added. The reaction was kept under reflux for $48 \mathrm{~h}$ (complete consumption of $\mathbf{4} / \mathbf{5}$ as judged by GC-MS). The solvent was then evaporated under reduced pressure to give a dark viscous oil $\left({ }^{1} \mathrm{H}-\mathrm{NMR}\right.$ data of the crude were consistent with data of the benzylimine reported in the literature). The crude was dissolved in methanol $(35 \mathrm{~mL})$ and then $\mathrm{NaBH}_{4}$ (1.5 equiv) was added portionwise at $0^{\circ} \mathrm{C}$. The reaction mixture was stirred at $\mathrm{rt}$ for $6 \mathrm{~h}$ and then quenched with water $(10$ $\mathrm{mL}$ ). Methanol was removed under reduced pressure, the aqueous phase was extracted with AcOEt, the combined organics were dried over $\mathrm{Na}_{2} \mathrm{SO}_{4}$ and then concentrated to give a crude brown oil.

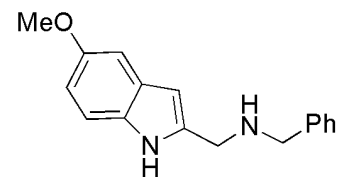

(c-Hex:AcOEt 8:2 $\rightarrow 1: 1$ ). Brown solid. Yield 79\% (two steps). M.p. $=77$ $78^{\circ} \mathrm{C} .{ }^{1} \mathrm{H}-\mathrm{NMR}\left(200 \mathrm{MHz}, \mathrm{CDCl}_{3}\right): \delta 3.75(\mathrm{~s}, 2 \mathrm{H}), 3.79(\mathrm{~s}, 3 \mathrm{H}), 3.89(\mathrm{~s}, 2 \mathrm{H})$, $6.23(\mathrm{~s}, 1 \mathrm{H}), 6.74(\mathrm{dd}, J=1.4,8.8 \mathrm{~Hz}, 1 \mathrm{H}), 6.97(\mathrm{~s}, 1 \mathrm{H}), 7.13-7.29(\mathrm{~m}, 7 \mathrm{H})$, $8.44(\mathrm{br}, 1 \mathrm{H}) ;{ }^{13} \mathrm{C}-\mathrm{NMR}\left(50 \mathrm{MHz}, \mathrm{CDCl}_{3}\right): \delta 45.8,52.7,55.8,100.7,102.2,111.4,111.6,127.4$, 128.3(2C), 128.6(2C), 128.7, 131.6, 137.2, 138.9, 154.1; GC-MS m/z 51(5), 65 (11), 77(9), 91(85), 106(56), 117(15), 165(100), 179(4), 191(44), 191, (2), 270(15); IR (nujol): v 3304(m), 2917(s), 2716(w), 1441(s), 1372(s), 1204(m), 1166(m) cm ${ }^{-1}$. Anal. calcd for $\left(\mathrm{C}_{17} \mathrm{H}_{18} \mathrm{NO}_{2}\right): \mathrm{C}, 76.66 ; \mathrm{H}, 6.81$; N, 10.52; Found: C, 76.60; H, 6.88; N, 10.55.

[Imine intermediate, ${ }^{1} \mathrm{H}-\mathrm{NMR}\left(200 \mathrm{MHz}, \mathrm{CDCl}_{3}\right): \delta 3.86(\mathrm{~s}, 3 \mathrm{H}), 4.87(\mathrm{~s}, 2 \mathrm{H}), 6.74(\mathrm{~s}, 1 \mathrm{H}), 6.95$ (dd, $J=2,6,9.2 \mathrm{~Hz}, 1 \mathrm{H}), 7.27-7.39(\mathrm{~m}, 7 \mathrm{H}), 8.34$ (s, 1H), 9.20 (br, 1H); IR $\left(\mathrm{CH}_{2} \mathrm{Cl}_{2}\right)$ : v 3406, 2924, 2853, 1636, 1483, 1457, $697 \mathrm{~cm}^{-1}$; GC-MS m/z 51(5), 65 (11), 77(9), 91(100), 117(15), 163(6), 191(44), 191, (49), 268(78)].

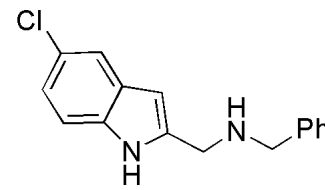

(c-Hex, washing). Yield 81\% (two steps). Brown solid. M.p. $=113-116^{\circ} \mathrm{C}$. ${ }^{1} \mathrm{H}-\mathrm{NMR}\left(300 \mathrm{MHz}, \mathrm{CDCl}_{3}\right): \delta 2.52(\mathrm{br}, 1 \mathrm{H}), 3.82,(\mathrm{~s}, 2 \mathrm{H}), 3.98(\mathrm{~s}, 2 \mathrm{H}), 6.31$ (s, 1H), $7.11(\mathrm{dd}, J=2.1,8.7 \mathrm{~Hz}, 1 \mathrm{H}), 7.21-7.35(\mathrm{~m}, 6 \mathrm{H}), 7.52(\mathrm{~s}, 1 \mathrm{H}), 8.88$

(br, 1H); ${ }^{13} \mathrm{C}-\mathrm{NMR}\left(75 \mathrm{MHz}, \mathrm{CDCl}_{3}\right): \delta 45.9,53.1,100.1,111.6,119.4,121.6,125.1,127.3,128.2$, 128.5, 129.5, 134.2, 138.8, 139.4; LC-ESI-MS 271 (M+1), 293 (M+Na); IR (nujol): v 3377, 2870, 
1462, 1376, $721 \mathrm{~cm}^{-1}$; Anal. calcd for $\left(\mathrm{C}_{16} \mathrm{H}_{15} \mathrm{ClN}_{2}\right): \mathrm{C}, 70.98 ; \mathrm{H}, 5.58 ; \mathrm{N}, 10.35$; Found: $\mathrm{C}, 70.89$; H, 5.53; N, 10.30.

[Imine intermediate, ${ }^{1} \mathrm{H}-\mathrm{NMR}\left(200 \mathrm{MHz}, \mathrm{CDCl}_{3}\right): \delta 4.79(\mathrm{~s}, 2 \mathrm{H}), 6.93(\mathrm{~s}, 1 \mathrm{H}), 7.12-7.40(\mathrm{~m}, 7 \mathrm{H})$, 7.57 (s, 1H), 8.32 (s, 1H), 9.22 (br, 1H); GC-MS m/z 51(6), 65(12), 91(100), 104(7), 117(11), 193(41), (195(43); IR (nujol): v 3085, 2868, 1537, 1451, 1314, 731, $689 \mathrm{~cm}^{-1}$ ].

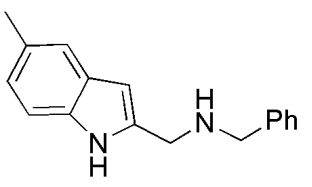

(c-Hex:AcOEt 1:1). Yield 77\% (two steps). Brown-red solid. $\mathrm{Mp}=79-81^{\circ} \mathrm{C}$. ${ }^{1} \mathrm{H}-\mathrm{NMR}\left(200 \mathrm{MHz}, \mathrm{CDCl}_{3}\right.$ ): $\delta 1.93$ (br, 1H), 2.45 (s, 3H), 3.82 (s, 2H), 3.97 $(\mathrm{s}, 2 \mathrm{H}), 6.29$ (s, 1H), 6.95 (d, $J=8.2 \mathrm{~Hz}, 1 \mathrm{H}), 7.25-7.38(\mathrm{~m}, 7 \mathrm{H}), 8.47$ (br, $1 \mathrm{H}) ;{ }^{13} \mathrm{C}-\mathrm{NMR}\left(50 \mathrm{MHz}, \mathrm{CDCl}_{3}\right): \delta 21.4,46.1,53.0,99.9,110.3,119.8,122.9,127.1(2 \mathrm{C})$, 128.1(2C), 128.5(2C), 128.7, 134.2, 137.5, 139.8; LC-ESI-MS 251 (M+1); IR (nujol): v 3266(w), 3128(m), 2856(m), 1549(w), 1452(s), 1208(w) cm ${ }^{-1}$; Anal. calcd for $\left(\mathrm{C}_{17} \mathrm{H}_{18} \mathrm{~N}_{2}\right): \mathrm{C}, 81.56 ; \mathrm{H}, 7.25$; N, 11.19; Found: C, 81.50; H, 7.19; N, 11.17.

[Imine intermediate, ${ }^{1} \mathrm{H}-\mathrm{NMR}\left(200 \mathrm{MHz}, \mathrm{CDCl}_{3}\right): \delta 2.45(\mathrm{~s}, 3 \mathrm{H}), 4.82(\mathrm{~s}, 2 \mathrm{H}), 6.74(\mathrm{~s}, 1 \mathrm{H}), 7.07-$ 7.44 (m, 8 H), 8.35 (s, 1H), 9.14 (br, 1H); GC-MS m/z 51(5), 65(12), 77(9), 91(92), 103(12), 177(21), 132(19), 144(19), 171(100), 220(10), 248(98); IR (neat): v 3404(s), 1635(s), 1451(m), $\left.1318(\mathrm{~m}), 1024(\mathrm{w}) \mathrm{cm}^{-1}\right]$.

(no-purification). Yield 91\% (two steps, purity $>95 \%$ by $\left.{ }^{1} \mathrm{H}-\mathrm{NMR}\right)$. Brown
viscous oil. ${ }^{1} \mathrm{H}-\mathrm{NMR}\left(200 \mathrm{MHz}, \mathrm{CDCl}_{3}\right): \delta 1.87(\mathrm{~s}, 1 \mathrm{H}), 3.80(\mathrm{~s}, 2 \mathrm{H}), 3.83(\mathrm{~s}, 2 \mathrm{H})$, $6.05(\mathrm{~s}, 1 \mathrm{H}), 6.15(\mathrm{q}, J=2.8 \mathrm{~Hz}, 1 \mathrm{H}), 6.75(\mathrm{dd}, J=2.2,4.0, \mathrm{~Hz}, 1 \mathrm{H}), 7.29-7.32(\mathrm{~m}, 5 \mathrm{H}), 8.62(\mathrm{br}$, $1 \mathrm{H}) ;{ }^{13} \mathrm{C}-\mathrm{NMR}\left(75 \mathrm{MHz}, \mathrm{CDCl}_{3}\right): \delta 46.0,53.3,106.7,108.3,117.5,127.4,128.4(2 \mathrm{C}), 128.8(2 \mathrm{C})$, 130.4, 140.1; LC-ESI-MS 187 (M+1); IR (nujol): v 3190(s), 3061(m), 1494(w), 1098(m) cm Anal. calcd for $\left(\mathrm{C}_{12} \mathrm{H}_{14} \mathrm{~N}_{2}\right)$ : C, 77.38 H, 7.58; N, 15.04; Found: C, 77.40; H, 7.55 N, 15.01.

[Imine intermediate, ${ }^{1} \mathrm{H}-\mathrm{NMR}\left(200 \mathrm{MHz}, \mathrm{CDCl}_{3}\right): \delta 4.72(\mathrm{~s}, 2 \mathrm{H}), 6.26(\mathrm{dd}, J=2,4,3.6 \mathrm{~Hz}, 1 \mathrm{H})$, 6.56 (dd, $J=1.4,3.6 \mathrm{~Hz}, 1 \mathrm{H}), 6.92$ (br, 1H), 7.31-7.36 (m, 5H), 8.14 (s, 1H); GC-MS m/z 51(5), 65(21), 79(22), 91(100), 106(15), 177(33), 156(10), 184(72); IR (neat): v 1734(s), 1637(s), 1027 (m) $\left.\mathrm{cm}^{-1}\right]$.

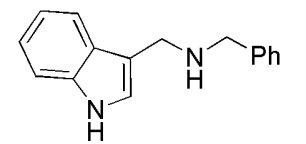

(washing with $c$-Hex). Yield 90\% (two steps). Pale yellow solid. $\mathrm{Mp}=89-90^{\circ} \mathrm{C}$. ${ }^{1} \mathrm{H}-\mathrm{NMR}\left(200 \mathrm{MHz}, \mathrm{CDCl}_{3}\right): \delta 1.72$ (br, 1H), 3.93 (s, 2H), $4.01(\mathrm{~s}, 2 \mathrm{H}), 7.11(\mathrm{~s}$, 1H), 7.16-7.40 (m, 8H), 7.69 (d, $J=7.4 \mathrm{~Hz}, 1 \mathrm{H}), 8.39$ (br, $1 \mathrm{H}) ;{ }^{13} \mathrm{C}-\mathrm{NMR}\left(75 \mathrm{MHz}, \mathrm{CDCl}_{3}\right): \delta$ $44.1,53.4,111.2$, 114.8, 118.8, 119.4, 122.0, 122.6, 126.9, 127.0, 128.2, 128.4, 136.3, 140.4; LC- 
ESI-MS 237 (M+1); IR (nujol): v 3317(w), 2952(s), 2854(s), 1456(m), 1379(m), 1247(w) cm ${ }^{-1}$; Anal. calcd for $\left(\mathrm{C}_{16} \mathrm{H}_{16} \mathrm{~N}_{2}\right)$ : C, 81.32; H, 6.82; N, 11.85; Found: C, 81.30; H, 6.78; N, 11.87.

[Imine intermediate, ${ }^{1} \mathrm{H}-\mathrm{NMR}\left(300 \mathrm{MHz}, \mathrm{CDCl}_{3}\right): \delta 4.86(\mathrm{~s}, 2 \mathrm{H}), 7.23-7.45(\mathrm{~m}, 10 \mathrm{H}), 8.37-8.40$ (m, 1H), 8.61 (s, 1H); IR (nujol): v 3023(m), 2931 (s), 1624(s), $\left.1460(\mathrm{~m}), 810(\mathrm{w}) \mathrm{cm}^{-1}\right]$.

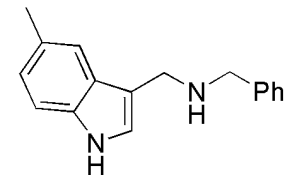

Yield 98\% (two steps). Pale-yellow solid. $\mathrm{Mp}=90-93^{\circ} \mathrm{C} .{ }^{1} \mathrm{H}-\mathrm{NMR}(200 \mathrm{MHz}$, $\left.\mathrm{CDCl}_{3}\right): \delta 1.64(\mathrm{~s}, 1 \mathrm{H}), 2.48(\mathrm{~s}, 3 \mathrm{H}), 3.91-4.00(\mathrm{~m}, 4 \mathrm{H}), 7.05(\mathrm{~d}, J=8.0 \mathrm{~Hz}, 1 \mathrm{H})$, $7.13(\mathrm{~s}, 1 \mathrm{H}), 7.28-7.43(\mathrm{~m}, 7 \mathrm{H}), 8.03(\mathrm{br}, 1 \mathrm{H}) ;{ }^{13} \mathrm{C}-\mathrm{NMR}\left(75 \mathrm{MHz}, \mathrm{CDCl}_{3}\right): \delta$ 21.5, 43.9, 53.2, 110.8, 118.4, 122.8, 123.7, 126.9, 127.0, 128.3, 128.4, 128.5, 128.7, 134.6, 140.2; LC-ESI-MS 251 (M+1); IR (nujol): v 2952(s), 2925(s), 2854(s), 1500(w), 1456(s), 1379(m), 1352(m), 1110(m) cm $\mathrm{cm}^{-1}$; Anal. calcd for $\left(\mathrm{C}_{17} \mathrm{H}_{18} \mathrm{~N}_{2}\right)$ : $\mathrm{C}, 81.56 ; \mathrm{H}, 7.25 ; \mathrm{N}, 11.19$; Found: $\mathrm{C}, 81.53$; $\mathrm{H}, 7.21 ; \mathrm{N}, 11.18$.

[Imine intermediate, ${ }^{1} \mathrm{H}-\mathrm{NMR}\left(200 \mathrm{MHz}, \mathrm{CDCl}_{3}\right): \delta 2.48(\mathrm{~s}, 3 \mathrm{H}), 4.86(\mathrm{~s}, 2 \mathrm{H}), 7.08(\mathrm{dd}, J=1.6,6.8$ $\mathrm{Hz}, 1 \mathrm{H}), 7.22-7.45(\mathrm{~m}, 8 \mathrm{H}), 8.17(\mathrm{~s}, 1 \mathrm{H}), 8.58$ (s, 1H); LC-ESI-MS: $250(\mathrm{M}+1)$; IR (nujol): $v$ 3025(m), $\left.2929(\mathrm{~s}), 1622(\mathrm{~s}), 1463(\mathrm{~m}), 800(\mathrm{w}) \mathrm{cm}^{-1}\right]$.

\section{Preparation of carbonic acid $(E)-4$-[benzyl-(1H-indol-2-yl-methyl)-amino]-but-2-enyl ethyl} esters.

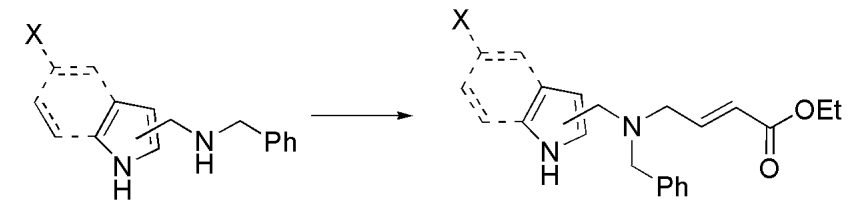

To a solution of amine ( $2 \mathrm{mmol}, 1.0$ equiv) and $\mathrm{Et}_{3} \mathrm{~N}$ (1.2 equiv) in dry THF (25 mL), ethyl 4bromocrotonate ( 2.0 equiv) was added at $0^{\circ} \mathrm{C}$. The ice bath was removed and the reaction mixture was stirred at $\mathrm{rt}$ for $4 \mathrm{~h}$. Finally, the mixture was filtered and solid residue washed with $\mathrm{Et}_{2} \mathrm{O}$. Evaporation of the volatiles under reduced pressure and subsequent purification by flashchromatography.

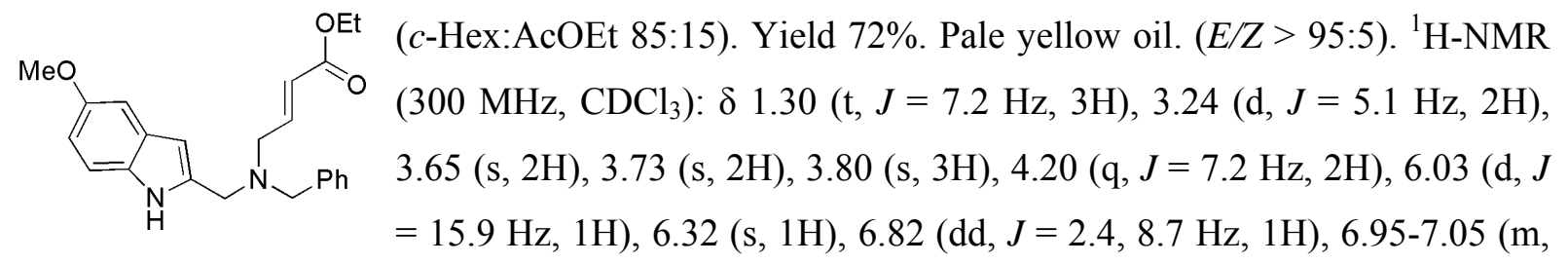

2H), 7.22-7.63 (m, 6H), 8.24 (br, $1 \mathrm{H}) ;{ }^{13} \mathrm{C}-\mathrm{NMR}\left(75 \mathrm{MHz}, \mathrm{CDCl}_{3}\right): \delta$ 14.2, 51.3, 54.2, 55.8, 58.2, $60.5,101.3,111.6,119.5,121.9,123.4,125.3,127.5,128.5(2 \mathrm{C}), 128.8(2 \mathrm{C}), 129.5,134.3,137.6$, 
137.8, 145.6, 166.2; LC-ESI-MS: 379 (M+1); IR (neat): v 3086(s), 2992(w), 2708(w), 1744(s), 1583(m), 1488(s), 1277(s) cm $\mathrm{cm}^{-1}$; Anal. calcd for $\left(\mathrm{C}_{23} \mathrm{H}_{26} \mathrm{~N}_{2} \mathrm{O}_{3}\right)$ : C, 72.99; H, 6.92; N, 7.40; Found: C, 72.91; H, 6.89; N, 7.37.

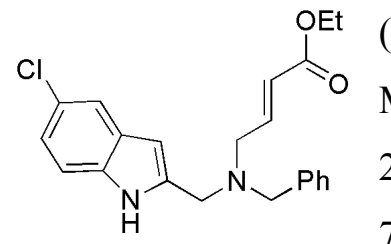

(c-Hex:AcOEt 8:2). Yield 85\%. Pale yellow oil. (E/Z > 95:5). ${ }^{1} \mathrm{H}-\mathrm{NMR}(200$ $\left.\mathrm{MHz}, \mathrm{CDCl}_{3}\right): \delta 1.30(\mathrm{t}, J=7.2 \mathrm{~Hz}, 3 \mathrm{H}), 3.26(\mathrm{~s}, 2 \mathrm{H}), 3.62(\mathrm{~s}, 2 \mathrm{H}), 3.74(\mathrm{~s}$, 2H), 4.19 (q, $J=7.2 \mathrm{~Hz}, 2 \mathrm{H}), 6.02(\mathrm{~d}, J=15.8 \mathrm{~Hz}, 1 \mathrm{H}), 5.32(\mathrm{~s}, 1 \mathrm{H}), 6.95-$ $7.09(\mathrm{~m}, 1 \mathrm{H}), 7.11-7.13(\mathrm{~m}, 1 \mathrm{H}), 7.22-7.35(\mathrm{~m}, 6 \mathrm{H}), 7.50(\mathrm{~s}, 1 \mathrm{H}), 8.19$ (br, $1 \mathrm{H}) ;{ }^{13} \mathrm{C}-\mathrm{NMR}\left(75 \mathrm{MHz}, \mathrm{CDCl}_{3}\right): \delta 14.2,51.2,54.3,58.4,60.5,101.3,111.6,119.5,121.9,123.4$, $125.3,127.5,128.5(2 \mathrm{C}), 128.8(2 \mathrm{C}), 129.5,134.3,137.6,137.8,145.3,166.9$; LC-ESI-MS: 383 $(\mathrm{M}+1), 406(\mathrm{M}+\mathrm{Na}), 421(\mathrm{M}+\mathrm{K})$; IR (neat): v 3646(w), 3367(s), 3188(m), $3026(\mathrm{~m}), 2977(\mathrm{~s})$, 2804(s), 2714(w), 1706(s), 1657(m), 1576(s), 1452(m) cm $\mathrm{cm}^{-1}$ Anal. calcd for $\left(\mathrm{C}_{22} \mathrm{H}_{23} \mathrm{ClN}_{2} \mathrm{O}_{2}\right): \mathrm{C}$, 69.01; H, 6.05; N, 7.32; Found: C, 68.94; H, 6.00; N, 7.33.

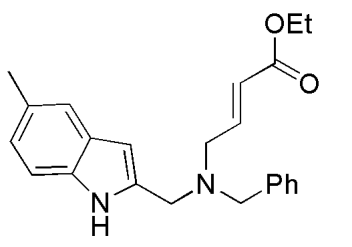

(c-Hex:AcOEt 85:15). Yield 92\%. Viscous pink oil. (E/Z > 95:5). ${ }^{1} \mathrm{H}-\mathrm{NMR}$ $\left(200 \mathrm{MHz}, \mathrm{CDCl}_{3}\right): \delta 1.33(\mathrm{t}, J=7.4 \mathrm{~Hz}, 3 \mathrm{H}), 2.43(\mathrm{~s}, 3 \mathrm{H}), 3.24(\mathrm{~d}, J=5.0$ $\mathrm{Hz}, 2 \mathrm{H}), 3.64$ (s, 2H), 3.73 (s, 2H), 4.20 (q, $J=7.4 \mathrm{~Hz}, 2 \mathrm{H}), 6.05$ (d, $J=13.8$ $\mathrm{Hz}, 1 \mathrm{H}), 6.30(\mathrm{~s}, 1 \mathrm{H}), 6.93-7.06(\mathrm{~m}, 1 \mathrm{H}), 7.24-7.41(\mathrm{~m}, 8 \mathrm{H}), 8.25$ (br, 1H); ${ }^{13} \mathrm{C}-\mathrm{NMR}\left(50 \mathrm{MHz}, \mathrm{CDCl}_{3}\right): \delta$ 14.2, 21.4, 51.3, 54.1, 58.2, 60.5, 101.3, 110.4, 119.8, 122.0123 .2 , 125.0 127.4, 128.5(2C), 128.9(2C), 132.8, 136.0, 138.4, 142.5, 145.7, 166.2; LC-ESI-MS: 363 $(\mathrm{M}+1), 385(\mathrm{M}+\mathrm{Na})$; IR (neat): v 3399(s), 3085(w), 3052(m), 2981(s), 2805(s), 1709(s), 1659(m), $1599(\mathrm{~m}), 1455(\mathrm{~s}) \mathrm{cm}^{-1}$; Anal. calcd for $\left(\mathrm{C}_{23} \mathrm{H}_{26} \mathrm{~N}_{2} \mathrm{O}_{2}\right)$ : C, 76.21; H, 7.23; N, 7.73; Found: C, 76.25; H, 7.18; N, 7.71.

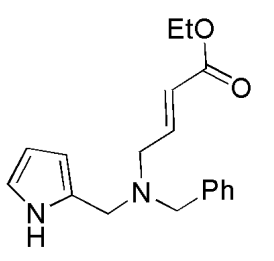

(c-Hex:AcOEt 90:10). Yield 73\%. Pale yellow oil. (E/Z > 95:5). ${ }^{1} \mathrm{H}-\mathrm{NMR}(300$ $\left.\mathrm{MHz}, \mathrm{CDCl}_{3}\right): \delta 1.33(\mathrm{t}, J=7.2 \mathrm{~Hz}, 3 \mathrm{H}), 3.23(\mathrm{~d}, J=6.0 \mathrm{~Hz}, 2 \mathrm{H}), 3.63(\mathrm{~s}, 2 \mathrm{H})$, $3.73(\mathrm{~s}, 2 \mathrm{H}), 4.24$ (q, $J=7.2 \mathrm{~Hz}, 2 \mathrm{H}), 6.01-6.07(\mathrm{~m}, 2 \mathrm{H}), 6.16(\mathrm{dd}, J=3.3,4.8$ $\mathrm{Hz}, 1 \mathrm{H}), 6.77-6.79(\mathrm{~m}, 1 \mathrm{H}), 7.00(\mathrm{dt}, J=6.2,13.8 \mathrm{~Hz}, 1 \mathrm{H}), 7.30-7.35(\mathrm{~m}, 5 \mathrm{H})$, 8.41 (br, $1 \mathrm{H}) ;{ }^{13} \mathrm{C}-\mathrm{NMR}\left(75 \mathrm{MHz}, \mathrm{CDCl}_{3}\right): \delta 14.2,50.8,54.1,58.1,60.4,107.6,108.1,117.4$, 123.1, 127.4, 128.1, 128.4(2C), 128.8(2C), 138.5, 145.9, 166.2; LC-ESI-MS: 299 (M+1); IR (neat): $v$ 3365(s), 3050(w), 2805(s), 1712(s), 1660(s), 1599(m), 1435(m) cm ${ }^{-1}$; Anal. calcd for $\left(\mathrm{C}_{18} \mathrm{H}_{22} \mathrm{~N}_{2} \mathrm{O}_{2}\right)$ : C, 72.46; H, 7.43; N, 9.39; Found: C, 72.44; H, 7.40; N, 9.35. 


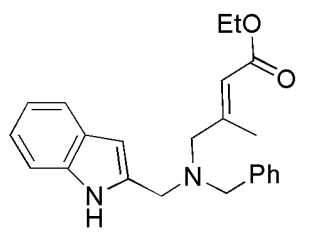

(E)-Ethyl 4-bromo-3-methylcrotonate ${ }^{2}$ was used for the synthesis. (cHex:AcOEt 90:10). Yield 45\%. Pale yellow oil. (E/Z > 95:5). ${ }^{1} \mathrm{H}-\mathrm{NMR}(200$ $\left.\mathrm{MHz}, \mathrm{CDCl}_{3}\right): \delta 1.30(\mathrm{t}, J=7.2 \mathrm{~Hz}, 3 \mathrm{H}), 2.18(\mathrm{~s}, 3 \mathrm{H}), 3.09(\mathrm{~s}, 2 \mathrm{H}), 3.59$ (s, 2H), 3.69 (s, 2H), 4.17 (q, $J=7.2 \mathrm{~Hz}, 2 \mathrm{H}), 6.03$ (s, 1H), 6.39 (s, 1H), 7.08-7.17 (m, 1H), 7.19-7.35 (m, 7H), $7.53(\mathrm{~d}, J=5.8 \mathrm{~Hz}, 1 \mathrm{H}), 8.21$ (br, $1 \mathrm{H}) ;{ }^{13} \mathrm{C}-\mathrm{NMR}\left(50 \mathrm{MHz}, \mathrm{CDCl}_{3}\right): \delta$ 14.3, 17.5, 51.2, 58.4, 59.7, 62.1, 101.9, 110.7, 117.3, 119.4, 119.7, 120.1, 121.3, 121.6, 127.3, 128.5(2C), 128.9(2C), 136.1, 138.3, 156.3, 166.5; LC-ESI-MS: $363(\mathrm{M}+1), 385(\mathrm{M}+\mathrm{Na})$; Anal. calcd for $\left(\mathrm{C}_{23} \mathrm{H}_{26} \mathrm{~N}_{2} \mathrm{O}_{2}\right)$ : C, 76.21; H, 7.23; N, 7.73; Found: C, 76.15; H, 7.20; N, 7.70.

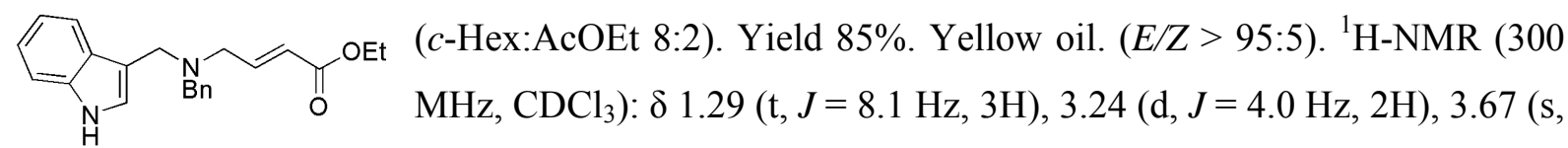
2H), $3.81(\mathrm{~s}, 2 \mathrm{H}), 4.19$ (q, $J=4.0 \mathrm{~Hz}, 2 \mathrm{H}), 6.05(\mathrm{dd}, J=1.8,15.9 \mathrm{~Hz}, 1 \mathrm{H}), 7.00-7.11(\mathrm{~m}, 1 \mathrm{H}), 7.15-$ $7.41(\mathrm{~m}, 9 \mathrm{H}), 7.74$ (d, $J=7.5 \mathrm{~Hz}, 1 \mathrm{H}), 8.01$ (br, 1H); ${ }^{13} \mathrm{C}-\mathrm{NMR}\left(75 \mathrm{MHz}, \mathrm{CDCl}_{3}\right): \delta$ 14.3, 49.6, 54.2 , 58.4, 60.3, 111.0, 113.3, 119.5, 119.7, 122.0, 122.6, 123.4, 126.9, 127.6(2C), 128.2(2C), 128.8, 136.4, 139.3, 147.0, 166.4; LC-ESI-MS: 363 (M+1); IR (neat): v 3416(s), 3025(m), 2904(m), 2786(s), 1703(s), 1659(m), 1615(w), 1301(s), 1037(m) cm $\mathrm{cm}^{-1}$ : Anal. calcd for $\left(\mathrm{C}_{22} \mathrm{H}_{24} \mathrm{~N}_{2} \mathrm{O}_{2}\right)$ : C, 75.83; H, 6.94; N, 8.04; Found: C, 75.80; H, 6.92; N, 8.03.

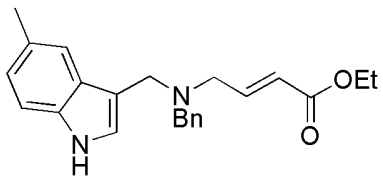

(c-Hex:AcOEt 8:2). Yield 88\%. Brown viscous oil. (E/Z > 95:5). ${ }^{1} \mathrm{H}-$ NMR (300 MHz, $\left.\mathrm{CDCl}_{3}\right): \delta 1.30(\mathrm{t}, J=7.2 \mathrm{~Hz}, 3 \mathrm{H}), 2.47(\mathrm{~s}, 3 \mathrm{H}), 3.25$ $(\mathrm{dd}, J=1.5,6.0 \mathrm{~Hz}, 2 \mathrm{H}), 3.66(\mathrm{~s}, 2 \mathrm{H}), 3.78(\mathrm{~s}, 2 \mathrm{H}), 4.20(\mathrm{q}, J=7.2 \mathrm{~Hz}$, 2H), 6.08 (dt, $J=1.5,15.6 \mathrm{~Hz}, 1 \mathrm{H}), 7.01-7.08(\mathrm{~m}, 2 \mathrm{H}), 7.11$ (d, $J=2.1 \mathrm{~Hz}, 1 \mathrm{H}), 7.23-7.42$ (m, 7H), 7.99 (br, $1 \mathrm{H}) ;{ }^{13} \mathrm{C}-\mathrm{NMR}\left(75 \mathrm{MHz}, \mathrm{CDCl}_{3}\right): \delta 14.2,21.5,49.3,54.2,58.3,60.3,110.6,119.3,122.6$, 123.5, 123.6, 125.3, 126.9, 127.9(2C), 128.2(2C), 128.6, 128.8, 134.7, 139.4, 147.0, 166.4; LC-ESIMS: 363 (M+1); IR (neat): v 3389(s), 3024(m), 2983(m), 2799(m), 1711(s), 1653(m), 1451(m), 1367(m), 1176(m) cm ${ }^{-1}$; Anal. calcd for $\left(\mathrm{C}_{23} \mathrm{H}_{26} \mathrm{~N}_{2} \mathrm{O}_{2}\right)$ : C, 76.21; H, 7.23; N, 7.73; Found: C, 76.18; H, 7.16; N, 7.70.

Preparation of $(E)-4-[b e n z y l-(1 H$-indol-2-yl-methyl)-amino]-but-2-enyl alcohol.
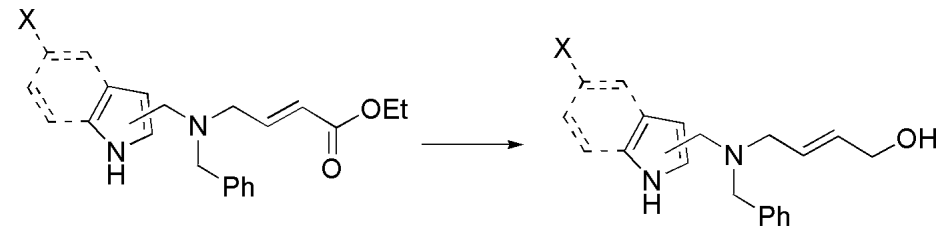
The ethyl ester (1.5 mmol, 1.0 equiv) was dissolved in dry toluene $(20 \mathrm{~mL})$ and then DIBALH (1.0 $\mathrm{M}$ in hexane, 2.5 equiv) was added dropwise at $-78^{\circ} \mathrm{C}$. The reaction was stirred at $-78^{\circ} \mathrm{C}$ for $1 \mathrm{~h}$ (complete consumption of the ester was judged by TLC) and then quenched with $0.5 \mathrm{~mL}$ of AcOH: $\mathrm{H}_{2} \mathrm{O}$ (1:1) mixture. The organics were washed with $\mathrm{H}_{2} \mathrm{O}$ concentrated under reduced pressure and purified by flash chromatography.

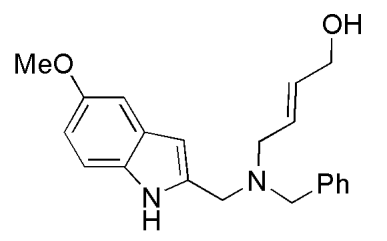

$\left(\mathrm{CH}_{2} \mathrm{Cl}_{2}: \mathrm{MeOH} 9: 1\right)$. Yield 91\%. Brown viscous oil. ${ }^{1} \mathrm{H}-\mathrm{NMR}(200 \mathrm{MHz}$, $\left.\mathrm{CDCl}_{3}\right): \delta 2.50(\mathrm{br}, 1 \mathrm{H}), 3.14(\mathrm{~d}, J=3.4 \mathrm{~Hz}, 1 \mathrm{H}), 3.66(\mathrm{~s}, 2 \mathrm{H}), 3.75(\mathrm{~s}, 2 \mathrm{H})$, $3.84(\mathrm{~s}, 3 \mathrm{H}), 4.11-4.18(\mathrm{~m}, 1 \mathrm{H}), 5.79(\mathrm{~s}, 1 \mathrm{H}), 6.30(\mathrm{~s}, 1 \mathrm{H}), 6.80(\mathrm{dd}, J=3.4$, $8.2 \mathrm{~Hz}, 1 \mathrm{H}), 7.03$ (s, 3H), 7.23-7.37 (m, 7H), $8.56(\mathrm{br}, 1 \mathrm{H}) ;{ }^{13} \mathrm{C}-\mathrm{NMR}(50$ $\left.\mathrm{MHz}, \mathrm{CDCl}_{3}\right): \delta 51.0,55.1,55.9,58.0,63.1,101.5,102.1,111.4,111.6,127.3(2 \mathrm{C}), 128.4(2 \mathrm{C})$, 127.9, 128.8, 129.1, 131.2, 133.1, 136.7, 137.9, 154.1; LC-ESI-MS: 336 (M+1); IR (nujol): v 3605(w), 3453(m), 3318(m), 3048(s), 2983(m), 2837(s), 1614(m), 1587(s), 1479(s), 1263(s) cm Anal. calcd for $\left(\mathrm{C}_{21} \mathrm{H}_{24} \mathrm{~N}_{2} \mathrm{O}_{2}\right)$ : C, 74.97; H, 7.19; N, 8.33; Found: C, 74.90; H, 7.12; N, 8.30.

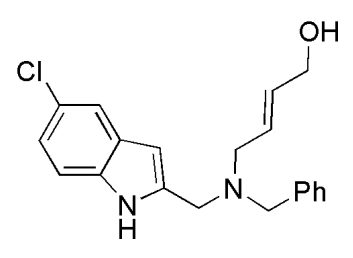

$\left(\mathrm{CH}_{2} \mathrm{Cl}_{2}: \mathrm{MeOH}\right.$ 9:1). Yield 98\%. Brown viscous oil. ${ }^{1} \mathrm{H}-\mathrm{NMR}(200 \mathrm{MHz}$ $\left.\mathrm{CDCl}_{3}\right): \delta 3.13(\mathrm{~s}, 2 \mathrm{H}), 3.65(\mathrm{~s}, 2 \mathrm{H}), 3.75(\mathrm{~s}, 2 \mathrm{H}), 4.13(\mathrm{~s}, 2 \mathrm{H}), 5.78-5.80(\mathrm{~m}$, $2 \mathrm{H}), 6.31(\mathrm{~s}, 1 \mathrm{H}), 7.05-7.17(\mathrm{~m}, 1 \mathrm{H}), 7.20-7.35(\mathrm{~m}, 7 \mathrm{H}), 7.40(\mathrm{~s}, 1 \mathrm{H}), 7.48$ (br, $1 \mathrm{H}) ;{ }^{13} \mathrm{C}-\mathrm{NMR}\left(75 \mathrm{MHz}, \mathrm{CDCl}_{3}\right): \delta 50.7,55.1,57.9,62.9,101.3,111.7$, 119.4, 121.7, 125.1, 125.3, 127.4, 127.5, 128.2, 128.4, 129.0, 129.1, 129.4, 133.3, 134.4, 137.5; LCESI-MS: $341(\mathrm{M}+1), 363(\mathrm{M}+\mathrm{Na})$; IR (neat): v 3556(m), 2415(s), 3026(m), 2918(s), 2815(s), 1717(m), 1576(s), 1490(s), 1144(w) $\mathrm{cm}^{-1}$; Anal. calcd for $\left(\mathrm{C}_{20} \mathrm{H}_{21} \mathrm{ClN}_{2} \mathrm{O}\right): \mathrm{C}, 70.48 ; \mathrm{H}, 6.21 ; \mathrm{N}$, 8.22; Found: C, 70.45; H, 6.15; N, 8.15.

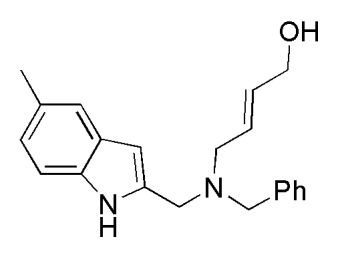

$\left(\mathrm{CH}_{2} \mathrm{Cl}_{2}: \mathrm{MeOH}\right.$ 9:1). Yield 94\%. Brown viscous oil. ${ }^{1} \mathrm{H}-\mathrm{NMR}(200 \mathrm{MHz}$, $\left.\mathrm{CDCl}_{3}\right): \delta 2.44(\mathrm{~s}, 3 \mathrm{H}), 3.23(\mathrm{~s}, 2 \mathrm{H}), 3.80(\mathrm{~s}, 2 \mathrm{H}), 3.90(\mathrm{~s}, 2 \mathrm{H}), 4.14(\mathrm{~s}, 2 \mathrm{H})$, 5.80-5.89 (m, 2H), $6.34(\mathrm{~s}, 1 \mathrm{H}), 6.99(\mathrm{~d}, J=8.0 \mathrm{~Hz}, 1 \mathrm{H}), 7.25-7.40(\mathrm{~m}, 8 \mathrm{H})$, 9.45 (br, $1 \mathrm{H}) ;{ }^{13} \mathrm{C}-\mathrm{NMR}\left(75 \mathrm{MHz}, \mathrm{CDCl}_{3}\right): \delta 21.7,49.7,53.3,55.9,62.1$, 105.6, 112.0, 118.9, 120.3, 124.9, 127.7(2C), 128.0(2C), 129.4, 129.7, 130.4, 131.2, 131.4, 136.1, 140.6; LC-ESI-MS: 321 (M+1); IR (neat): v 3245(s), 3031(s), 1722(s), 1554(m), 1452(s), 1315, (w), $1009(\mathrm{~m}) \mathrm{cm}^{-1}$; Anal. calcd for $\left(\mathrm{C}_{21} \mathrm{H}_{24} \mathrm{~N}_{2} \mathrm{O}\right)$ : C, 78.71; H, 7.55; N, 8.74; Found: C, 78.65; H, $7.49 ; \mathrm{N}, 8.70$. 


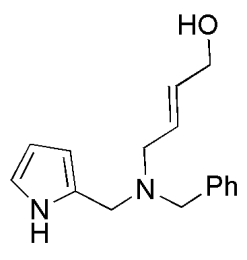

$\left(\mathrm{CH}_{2} \mathrm{Cl}_{2}: \mathrm{MeOH}\right.$ 95:5). Yield 41\%. Pale brown oil. ${ }^{1} \mathrm{H}-\mathrm{NMR}\left(300 \mathrm{MHz}, \mathrm{CDCl}_{3}\right): \delta$ 2,20 (br, 1H), 3.11 (d, $J=4.8 \mathrm{~Hz}, 2 \mathrm{H}), 3.63$ (s, 4H), 4.13 (s, 2H), 5.76 (d, J = 3.3, $\mathrm{Hz}, 2 \mathrm{H}), 6.04(\mathrm{~s}, 1 \mathrm{H}), 6.12(\mathrm{~s}, 1 \mathrm{H}), 6.47-6-76(\mathrm{~m}, 1 \mathrm{H}), 7.26-7-34(\mathrm{~m}, 5 \mathrm{H}), 8.70$ (br, $1 \mathrm{H}) ;{ }^{13} \mathrm{C}-\mathrm{NMR}\left(50 \mathrm{MHz}, \mathrm{CDCl}_{3}\right): \delta 50.5,54.7,57.8,62.9,108.0,108.3$, 118.0, 126.9, 127.6, 128.5(2C), 128.6, 129.4(2C), 133.9, 136.9; LC-ESI-MS: 257 (M+1); IR (neat): $v$ 3404(s), $2922(\mathrm{~s}), 2852(\mathrm{~m}), 1639(\mathrm{~m}), 1455(\mathrm{~m}) \mathrm{cm}^{-1}$; Anal. calcd for $\left(\mathrm{C}_{16} \mathrm{H}_{20} \mathrm{~N}_{2} \mathrm{O}\right): \mathrm{C}, 74.97 ; \mathrm{H}$, 7.86; N, 10.93; Found: C, 74.95; H, 7.85; N, 10.90.

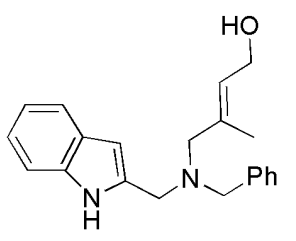

(c-Hex:AcOEt 70:30). Yield 37\%. Viscous yellow oil. (E/Z $>95: 5) .{ }^{1} \mathrm{H}-\mathrm{NMR}$ $\left(200 \mathrm{MHz}, \mathrm{CDCl}_{3}\right): \delta 1.74(\mathrm{~s}, 3 \mathrm{H}), 3.02(\mathrm{~s}, 2 \mathrm{H}), 3.60(\mathrm{~s}, 2 \mathrm{H}), 3.69(\mathrm{~s}, 2 \mathrm{H}), 4.18$ $(\mathrm{d}, J=7.8 \mathrm{~Hz}, 2 \mathrm{H}), 5.67$ (t, $J=5.8 \mathrm{~Hz}, 1 \mathrm{H}), 6.37$ (s, 1H), 7.05-7.19 (m, 2H), 7.22-7.37 (m, 6H), 7.56 (d, J=7.2 Hz, 1H), 8.35 (br, 1H); ${ }^{13} \mathrm{C}-\mathrm{NMR}(50 \mathrm{MHz}$, $\left.\mathrm{CDCl}_{3}\right): \delta 15.2,51.1,58.3,59.2,62.2,101.7,110.7,119.6,120.1,121.5,127.2,128.4,(2 \mathrm{C})$, 128.5(2C), 129.0, 132.8, 136.0, 136.7, 138.5; LC-ESI-MS: $321(\mathrm{M}+1)$; Anal. calcd for $\left(\mathrm{C}_{21} \mathrm{H}_{24} \mathrm{~N}_{2} \mathrm{O}\right)$ : C, 78.71; H, 7.55; N, 8.74; Found: C, 78.62; H, 7.51; N, 8.71.

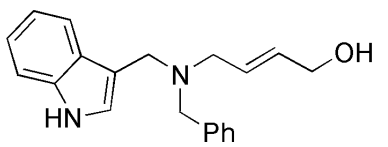

$\left(\mathrm{CH}_{2} \mathrm{C}_{2}\right.$ :EtOAc 95:5). Yield 65\%. Viscous yellow oil. ${ }^{1} \mathrm{H}-\mathrm{NMR}(200$ $\left.\mathrm{MHz}, \mathrm{CDCl}_{3}\right): \delta 1.82(\mathrm{br}, 1 \mathrm{H}), 3.13(\mathrm{~d}, J=3.0 \mathrm{~Hz}, 2 \mathrm{H}), 3.64(\mathrm{~s}, 2 \mathrm{H}), 3.80$ $(\mathrm{s}, 2 \mathrm{H}), 4.01(\mathrm{~s}, 2 \mathrm{H}), 5.79(\mathrm{~s}, 2 \mathrm{H}), 7.17-7.37(\mathrm{~m}, 9 \mathrm{H}), 7.75(\mathrm{~d}, J=7.0 \mathrm{~Hz}, 1 \mathrm{H}), 8.08(\mathrm{br}, 1 \mathrm{H}) ;{ }^{13} \mathrm{C}-$ NMR (50 MHz, $\left.\mathrm{CDCl}_{3}\right): \delta 49.4,55.2,58.1,63.4,111.0,113.6,119.4,119.8,122.0,123.4,126.8$, 127.8(2C), 128.1(2C), 128.9, 130.1, 131.6, 136.4, 139.7; LC-ESI-MS: 307 (M+1); IR (nujol): v 3423(s), 2926(s), 1653(s), 1380(m), 985(m), 572(m) cm ${ }^{-1}$; Anal. calcd for $\left(\mathrm{C}_{20} \mathrm{H}_{22} \mathrm{~N}_{2} \mathrm{O}\right): \mathrm{C}, 78.40$; H, 7.24; N, 9.14; Found: C, 78.35; H, 7.21; N, 9.13

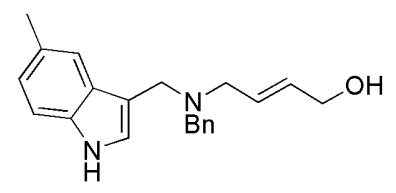

$\left(\mathrm{CH}_{2} \mathrm{Cl}_{2}\right.$ :EtOAc 95:5). Yield 55\%. Viscous yellow oil. ${ }^{1} \mathrm{H}-\mathrm{NMR}(200$ $\left.\mathrm{MHz} \mathrm{CDCl}_{3}\right): \delta 2.45(\mathrm{~s}, 3 \mathrm{H}), 3.28(\mathrm{~d}, J=4.4 \mathrm{~Hz}, 2 \mathrm{H}), 3.85(\mathrm{~s}, 2 \mathrm{H}), 4.01$ (s, 2H), $4.12(\mathrm{~s}, 2 \mathrm{H}), 5.80-5.8(\mathrm{~m}, 2 \mathrm{H}), 7.04(\mathrm{~d}, J=8.4 \mathrm{~Hz}, 1 \mathrm{H}), 7.30-7.43$ $(\mathrm{m}, 8 \mathrm{H}), 8.54$ (br, $1 \mathrm{H}) ;{ }^{13} \mathrm{C}-\mathrm{NMR}\left(75 \mathrm{MHz}, \mathrm{CDCl}_{3}\right): \delta$ 21.8, 22.6, 48.6, 54.2, 57.3, 63.0, 108.7, $111.4,118.8,119.0,124.1,128.2$, 128.5(2C), 128.8(2C), 129.3, 129.8, 130.1, 134.8, 135.3; LC-ESIMS: $321(\mathrm{M}+1)$; IR (neat): v 3302(s), 3044(m), 2908(m), 1576(s), 1436(m), 1263(w), 1093(w), $1008(w) \mathrm{cm}^{-1}$; Anal. calcd for $\left(\mathrm{C}_{21} \mathrm{H}_{24} \mathrm{~N}_{2} \mathrm{O}\right): \mathrm{C}, 78.71 ; \mathrm{H}, 7.55 ; \mathrm{N}, 8.74$; Found: $\mathrm{C}, 78.65 ; \mathrm{H}, 7.50$; $\mathrm{N}, 7.73$. 


\section{Preparation of the indolylacetate $\mathbf{1 b}$.}

To a solution of alcohol $(206 \mathrm{mg}, 0.67 \mathrm{mmol})$ in dry $\mathrm{CH}_{2} \mathrm{Cl}_{2}(8 \mathrm{~mL})$ were added $113 \mu \mathrm{L}(0.81$ mmol) of TEA and $9 \mathrm{mg}(10 \mathrm{~mol} \%)$ of DMAP. The solution was cooled to $0^{\circ} \mathrm{C}$, stirred for $10 \mathrm{~min}$ then $76 \mu \mathrm{L}(0.81 \mathrm{mmol})$ of acetic anhydride were added. The reaction mixture was allowed to stir at $\mathrm{rt}$ for $24 \mathrm{~h}$ then quenched with water $(5 \mathrm{~mL})$, extracted with AcOEt $(3 \times 7 \mathrm{~mL})$ and the collected organic phases washed with diluted $\mathrm{HCl}$. After evaporation under reduced pressure, $\mathbf{1 b}$ was purified by flash chromatography by using $c$-Hex:AcOEt 8:2 as the eluent mixture.

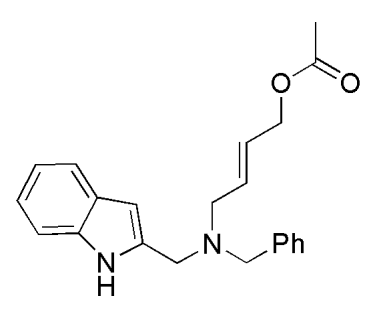

Pale yellow oil. Yield 75\%. ${ }^{1} \mathrm{H}-\mathrm{NMR}\left(200 \mathrm{MHz}, \mathrm{CDCl}_{3}\right): \delta 2.08(\mathrm{~s}, 3 \mathrm{H})$, $3.15(\mathrm{~d}, J=5.6 \mathrm{~Hz}, 2 \mathrm{H}), 3.67(\mathrm{~s}, 2 \mathrm{H}), 3.78(\mathrm{~s}, 2 \mathrm{H}), 4.56(\mathrm{~d}, J=5.0 \mathrm{~Hz}, 2 \mathrm{H})$, 5.80-5.85 (m, 2H), $6.38(\mathrm{~s}, 1 \mathrm{H}), 7.08-7.17(\mathrm{~m}, 1 \mathrm{H}), 7.17-7.40(\mathrm{~m}, 7 \mathrm{H}), 7.57$ $(\mathrm{d}, J=8.8 \mathrm{~Hz}, 1 \mathrm{H}), 9.62(\mathrm{br}, 1 \mathrm{H}) ;{ }^{13} \mathrm{C}-\mathrm{NMR}\left(50 \mathrm{MHz}, \mathrm{CDCl}_{3}\right): \delta 21.0$, $50.8,54.9,57.9,64.5,101.4,110.6,119.5,120.0,121.4,127.1,127.4$, 128.3, 128.4, 128.9, 132.1, 135.9, 136.5, 138.5, 170.8; LC-ESI-MS: 349 (M+1), $371(\mathrm{M}+\mathrm{Na})$; IR (neat): $v$ 3391(s), 3031(m), 2907(m), 2799(m), 1734(s), 1456(m), 1238(s), 1026(w) cm $\mathrm{cm}^{-1}$ Anal. calcd for $\left(\mathrm{C}_{22} \mathrm{H}_{24} \mathrm{~N}_{2} \mathrm{O}_{2}\right)$ : C, 75.83; H, 6.94; N, 8.04; Found: C, 75.80; H, 6.92; N, 8.01.

\section{Preparation of indolylcarbonates 1c-e/6a,b.}

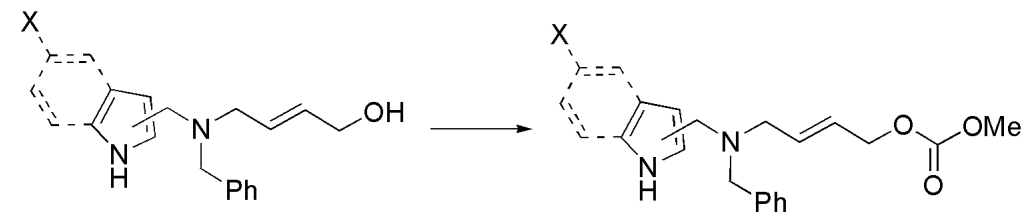

To a solution of alcohol (1.0 mmol, 1.0 equiv) and pyridine (2.0 equiv) in dry $\mathrm{Et}_{2} \mathrm{O}: \mathrm{CH}_{2} \mathrm{Cl}_{2}$ mixture $(1: 1,10 \mathrm{~mL})$, a solution of methyl chloroformate (1.5 equiv) dissolved in dry $\mathrm{Et}_{2} \mathrm{O}(1.0 \mathrm{~mL})$ was added dropwise at $0^{\circ} \mathrm{C}$. The reaction mixture was stirred for $24 \mathrm{~h}$ at $\mathrm{rt}$ then quenched with water $(5$ $\mathrm{mL})$, extracted with AcOEt $(3 \times 5 \mathrm{~mL})$, dried over $\mathrm{Na}_{2} \mathrm{SO}_{4}$, concentrated under reduced pressure and purified by flash chromatography.

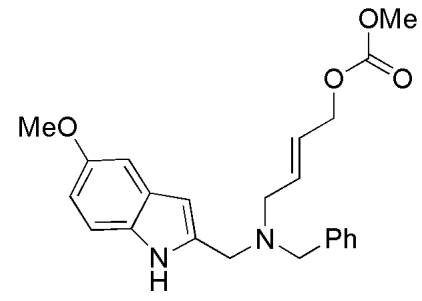

1c. $\left(\mathrm{CH}_{2} \mathrm{Cl}_{2}\right.$ :AcOEt 8:2). Yield 71\%. Yellow oil. ${ }^{1} \mathrm{H}-\mathrm{NMR}(200 \mathrm{MHz}$, $\left.\mathrm{CDCl}_{3}\right): \delta 3.16(\mathrm{~d}, J=5.6 \mathrm{~Hz}, 2 \mathrm{H}), 3.67(\mathrm{~s}, 2 \mathrm{H}), 3.76(\mathrm{~s}, 2 \mathrm{H}), 3.84(\mathrm{~s}$, $3 \mathrm{H}), 3.89(\mathrm{~s}, 3 \mathrm{H}), 4.66(\mathrm{~d}, J=5.6 \mathrm{~Hz}, 2 \mathrm{H}), 5.83-5.92(\mathrm{~m}, 2 \mathrm{H}), 6.35(\mathrm{~s}$, $1 \mathrm{H}), 6.85(\mathrm{dd}, J=2.2,8.8 \mathrm{~Hz}, 1 \mathrm{H}), 7.07$ (d, $J=2.6 \mathrm{~Hz}, 1 \mathrm{H}), 7.27-7.40$ $(\mathrm{m}, 6 \mathrm{H}), 8.37$ (br, $1 \mathrm{H}) ;{ }^{13} \mathrm{C}-\mathrm{NMR}\left(75 \mathrm{MHz}, \mathrm{CDCl}_{3}\right): \delta 50.9,54.8,55.9$, 58.0, 62.2, 67.8, 109.7, 111.6, 118.5, 121.4, 127.4, 128.0, 128.4, 128.6, 129.1, 133.4, 133.9, 134.4, 
137.7, 139.6, 154.1; LC-ESI-MS: $365(\mathrm{M}+1), 387(\mathrm{M}+\mathrm{Na}), 400(\mathrm{M}+\mathrm{K})$; IR (neat): v 3693(w), 3389(s), 3085(w), 3058(w), 3025(m), 2927(s), 1710(s), 1655(m), 1590(m), 1476(s), 1367(m) cm ${ }^{-1}$. Anal. calcd for $\left(\mathrm{C}_{23} \mathrm{H}_{26} \mathrm{~N}_{2} \mathrm{O}_{4}\right)$ : C, 70.03; H, 6.64; N, 7.10; Found: C, 70.00; H, 6.69; N, 7.11.

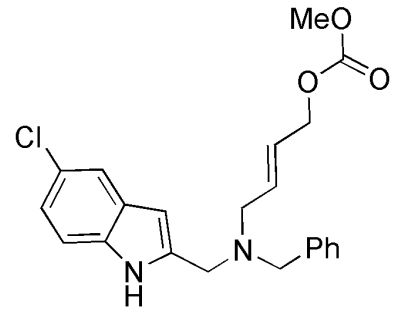

1d. (c-Hex:AcOEt 1:1). Yield 68\%. Yellow oil. ${ }^{1} \mathrm{H}-\mathrm{NMR}(200 \mathrm{MHz}$, $\left.\mathrm{CDCl}_{3}\right): \delta 3.06(\mathrm{~d}, J=5.7 \mathrm{~Hz}, 2 \mathrm{H}), 3.56(\mathrm{~s}, 2 \mathrm{H}), 3.66(\mathrm{~s}, 2 \mathrm{H}), 3.73(\mathrm{~s}$, $3 \mathrm{H}), 4.56(\mathrm{~d}, J=5.4 \mathrm{~Hz}, 2 \mathrm{H}), 5.76-5.82(\mathrm{~m}, 2 \mathrm{H}), 6.25(\mathrm{~s}, 1 \mathrm{H}), 7.06$ (dd, $J$ $=2.2,8.8 \mathrm{~Hz}, 1 \mathrm{H}), 7.19-7.29(\mathrm{~m}, 6 \mathrm{H}), 7.44(\mathrm{~s}, 1 \mathrm{H}), 8.39(\mathrm{br}, 1 \mathrm{H}) ;{ }^{13} \mathrm{C}-$ $\operatorname{NMR}\left(75 \mathrm{MHz}, \mathrm{CDCl}_{3}\right): \delta 50.7,54.8,58.0,60.4,67.8,101.0,111.6$, $119.4,121.6,125.1,126.9,127.3,128.4,128.9,129.5,132.6,134.2,138.1,138.3,155.6$. LC-ESIMS: $399(\mathrm{M}+1), 421(\mathrm{M}+\mathrm{Na}), 437(\mathrm{M}+\mathrm{K})$; IR (neat): $v$ 3432(s), 3031(m), 2874(s), 1744(s), 1457(m) $\mathrm{cm}^{-1}$. Anal. calcd for $\left(\mathrm{C}_{22} \mathrm{H}_{23} \mathrm{ClN}_{2} \mathrm{O}_{3}\right): \mathrm{C}, 66.24 ; \mathrm{H}, 5.81 ; \mathrm{N}, 7.02$; Found: $\mathrm{C}, 66.29 ; \mathrm{H}$, $5.80 ; \mathrm{N}, 6.98$.

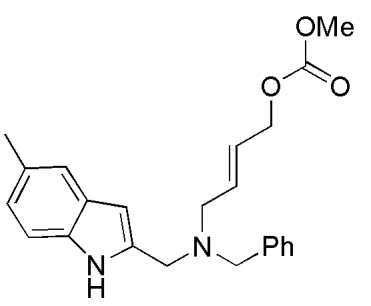

1e. $\left(\mathrm{CH}_{2} \mathrm{Cl}_{2}\right.$ :EtOAc 95:5). Yield 61\%. Pale yellow oil. ${ }^{1} \mathrm{H}-\mathrm{NMR}(300$ $\left.\mathrm{MHz}, \mathrm{CDCl}_{3}\right): \delta 2.42(\mathrm{~s}, 3 \mathrm{H}), 3.09(\mathrm{~d}, J=6.0 \mathrm{~Hz}, 2 \mathrm{H}), 3.60(\mathrm{~s}, 2 \mathrm{H}), 3.70$ (s, 2H), $3.80(\mathrm{~s}, 3 \mathrm{H}), 4.61(\mathrm{~d}, J=5.4 \mathrm{~Hz}, 2 \mathrm{H}), 5.70-5.90(\mathrm{~m}, 2 \mathrm{H}), 6.28$ (s, $1 \mathrm{H}), 6.97(\mathrm{dd}, J=1.2,8.1 \mathrm{~Hz}, 1 \mathrm{H}), 7.14-7.34(\mathrm{~m}, 7 \mathrm{H}), 8.29(\mathrm{br}, 1 \mathrm{H}) ;{ }^{13} \mathrm{C}-$ NMR (50 MHz, $\left.\mathrm{CDCl}_{3}\right): \delta 21.4,50.8,54.8(2 \mathrm{C}), 57.9,67.8,101.0,110.3$, 119.7, 123.0, 126.6, 127.1, 128.3(2C), 128.7(2C), 128.9(2C), 132.9, 134.3, 136.5, 138.6, 155.6. LCESI-MS: $379(\mathrm{M}+1)$; IR (neat): v 3395(s), 3030(m), 2878(s), 2807(s), 1734(s), 1582(w), 1495(m), $1250(\mathrm{~s}) \mathrm{cm}^{-1}$. Anal. calcd for $\left(\mathrm{C}_{23} \mathrm{H}_{26} \mathrm{~N}_{2} \mathrm{O}_{3}\right): \mathrm{C}, 72.99 ; \mathrm{H}, 6.92 ; \mathrm{N}, 7.40$; Found: $\mathrm{C}, 72.95 ; \mathrm{H}, 6.87$; N, 7.40.

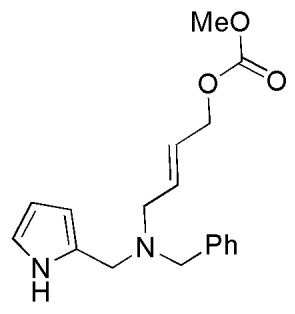

1f. (c-Hex:EtOAc 70:30). Yield 45\%. Pale yellow oil. ${ }^{1} \mathrm{H}-\mathrm{NMR}(300 \mathrm{MHz}$, $\left.\mathrm{CDCl}_{3}\right): \delta 3.09(\mathrm{~d}, J=5.7 \mathrm{~Hz}, 2 \mathrm{H}), 3.56(\mathrm{~d}, J=3.0 \mathrm{~Hz}, 4 \mathrm{H}), 3.79(\mathrm{~s}, 3 \mathrm{H}), 4.61$ $(\mathrm{d}, J=5.7 \mathrm{~Hz}, 2 \mathrm{H}), 5.74-5.81(\mathrm{~m}, 1 \mathrm{H}), 5.84-5.91(\mathrm{~m}, 1 \mathrm{H}), 6.03(\mathrm{~s}, 1 \mathrm{H}), 6.12$ $(\mathrm{dd}, J=2.7,5.4 \mathrm{~Hz}, 1 \mathrm{H}), 6.74(\mathrm{~s}, 1 \mathrm{H}), 7.27-7.33(\mathrm{~m}, 5 \mathrm{H}), 8.42(\mathrm{br}, 1 \mathrm{H}) ;{ }^{13} \mathrm{C}-$ $\operatorname{NMR}\left(75 \mathrm{MHz}, \mathrm{CDCl}_{3}\right): \delta 50.5,54.7,57.8,62.3,67.9,107.5,108.0,117.2$, 127.1, 128.3(2C), 129.0(2C), 129.9, 138.9, 155.7; LC-ESI-MS: $315(\mathrm{M}+1)$; Anal. calcd for $\left(\mathrm{C}_{18} \mathrm{H}_{22} \mathrm{~N}_{2} \mathrm{O}_{3}\right): \mathrm{C}, 68.77 ; \mathrm{H}, 7.05 ; \mathrm{N}, 8.91$; Found: $\mathrm{C}, 68.71 ; \mathrm{H}, 7.00 ; \mathrm{N}, 8.93$. 


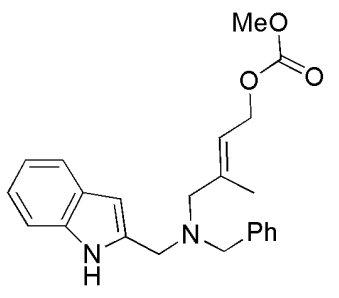

(c-Hex:AcOEt 95:5 $\rightarrow$ 90:10). Yield 93\%. Viscous yellow oil. $(E / Z>95: 5)$. ${ }^{1} \mathrm{H}-\mathrm{NMR}\left(200 \mathrm{MHz}, \mathrm{CDCl}_{3}\right): \delta 1.80(\mathrm{~s}, 3 \mathrm{H}), 3.01(\mathrm{~s}, 2 \mathrm{H}), 3.58(\mathrm{~s}, 2 \mathrm{H}), 3.67$ (s, 2H), $3.78(\mathrm{~s}, 3 \mathrm{H}), 4.72(\mathrm{~d}, J=7.0 \mathrm{~Hz}, 2 \mathrm{H}), 5.72(\mathrm{t}, J=7.2 \mathrm{~Hz}, 1 \mathrm{H}), 6.38$ (s, 1H), 7.05-7.20 (m, 2H), 7.23-7.37 (m, 6H), 7.57 (d, J=7.4 Hz, 1H), 8.32 (br, $1 \mathrm{H}) ;{ }^{13} \mathrm{C}-\mathrm{NMR}\left(50 \mathrm{MHz}, \mathrm{CDCl}_{3}\right): \delta 15.4,50.9,54.8,58.2,61.8,64.4$, 101.6, 110.6, 119.6, 120.1, 121.5, 121.6, 126.1, 127.4, 128.4(2C), 128.9(2C) , 129.2, 130.7, 140.4, 155.7; LC-ESI-MS: $379(\mathrm{M}+1), 401(\mathrm{M}+\mathrm{Na})$; IR (neat): v 3401, 3055, 3016, 2956, 2825, 1752, 1455, 1268, $1140 \mathrm{~cm}^{-1}$; Anal. calcd for $\left(\mathrm{C}_{23} \mathrm{H}_{26} \mathrm{~N}_{2} \mathrm{O}_{3}\right)$ : C, 72.99; H, 6.92; N, 7.40; Found: C, 72.95; $\mathrm{H}, 6.89 ; \mathrm{N}, 7.38$.

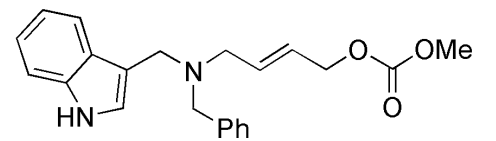

6a. $\left(\mathrm{CH}_{2} \mathrm{Cl}_{2}\right.$ :MeOH 95:5). Yield $84 \%$. Yellow viscous oil. ${ }^{1} \mathrm{H}-\mathrm{NMR}$ $\left(200 \mathrm{MHz}, \mathrm{CDCl}_{3}\right): \delta 3.13(\mathrm{~d}, J=5.4 \mathrm{~Hz}, 2 \mathrm{H}), 3.62(\mathrm{~s}, 2 \mathrm{H}), 3.69(\mathrm{~s}$, 2H), $3.78(\mathrm{~s}, 3 \mathrm{H}), 4.62(\mathrm{~d}, J=6.4 \mathrm{~Hz}, 2 \mathrm{H}), 5.74-5.84(\mathrm{~m}, 1 \mathrm{H}), 5.89-5.99(\mathrm{~m}, 1 \mathrm{H}), 7.09-7.39(\mathrm{~m}$, 9H), $7.73(\mathrm{~d}, J=7.2 \mathrm{~Hz}, 1 \mathrm{H}), 8.07(\mathrm{br}, 1 \mathrm{H}) ;{ }^{13} \mathrm{C}-\mathrm{NMR}\left(50 \mathrm{MHz}, \mathrm{CDCl}_{3}\right): \delta 49.1,54.7,57.9,60.3$, 68.1, 110.9, 113.2, 119.2, 119.7, 121.8, 123.4, 125.7, 126.7, 127.6(2C), 128.1(2C), 128.8, 134.2, 136.3, 139.6, 155.5. LC-ESI-MS: 365 (M+1), 387 (M+Na); IR (neat): v 3419(s), 3023(w), 2950(m), 2729(m), 1734(s), 1491(m), 1456(s), 1259(s), 1096(m), 736(s) $\mathrm{cm}^{-1}$. Anal. calcd for $\left(\mathrm{C}_{22} \mathrm{H}_{24} \mathrm{~N}_{2} \mathrm{O}_{3}\right)$ : C, 72.50; H, 6.64; N, 7.69; Found: C, 72.46; H, 6.56; N, 7.60.

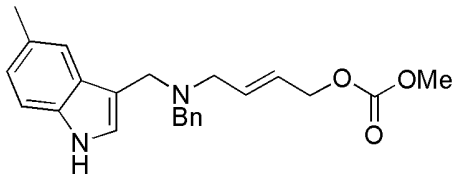

6b. $\left(\mathrm{CH}_{2} \mathrm{Cl}_{2}: \mathrm{MeOH} 95: 5\right)$. Yield 86\%. Brown viscous oil. ${ }^{1} \mathrm{H}-\mathrm{NMR}$ $\left(200 \mathrm{MHz}, \mathrm{CDCl}_{3}\right): \delta 2.47(\mathrm{~s}, 3 \mathrm{H}), 3.13(\mathrm{~d}, J=5.4 \mathrm{~Hz}, 2 \mathrm{H}), 3.62$ (s, $2 \mathrm{H}), 3.75(\mathrm{~s}, 2 \mathrm{H}), 3.79(\mathrm{~s}, 3 \mathrm{H}), 4.62(\mathrm{~d}, J=6.0 \mathrm{~Hz}, 2 \mathrm{H}), 5.70-6.01$ (m, 2H), $7.04(\mathrm{~d}, J=8.4 \mathrm{~Hz}, 1 \mathrm{H}), 7.10(\mathrm{~s}, 1 \mathrm{H}), 7.23-7.45(\mathrm{~m}, 7 \mathrm{H}), 7.94(\mathrm{br}, 1 \mathrm{H}) ;{ }^{13} \mathrm{C}-\mathrm{NMR}(50$ $\mathrm{MHz}_{\mathrm{CDCl}}$ ): $\delta 21.5,49.0,54.8,57.9,64.7,68.1,110.6,112.9,119.4,123.6,125.8,126.5,126.8$, 128.0(2C), 128.1(2C), 128.5, 129.0, 134.2, 134.7, 139.8, 155.6. LC-ESI-MS: 379 (M+1); IR (neat): v 3419(s), 3023(m), 2950(m), 2792(s), 1734(s), 1616(w), 1491(m), 1456(s), 1259(s), 736(m) cm $\mathrm{cm}^{-1}$. Anal. calcd for $\left(\mathrm{C}_{23} \mathrm{H}_{26} \mathrm{~N}_{2} \mathrm{O}_{3}\right)$ : C, 72.99; H, 6.92; N, 7.40; Found: C, 72.95; H, 6.90; N, 7.41.

\section{Preparation of indolylcarbonate $1 \mathrm{~h}$.}



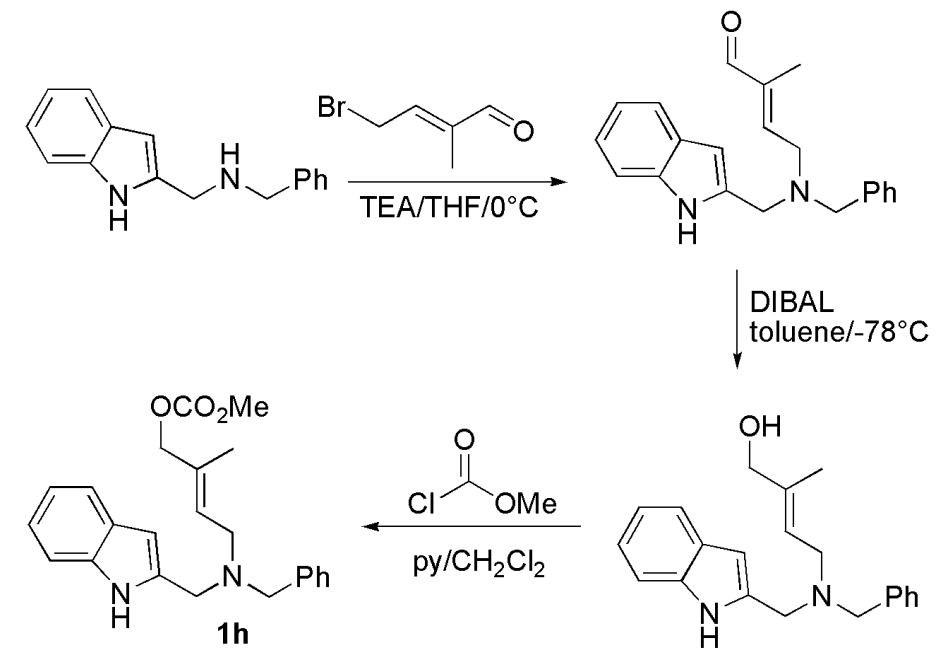

The synthesis of the indolyl-aldehyde was performed analogously to the previously described procedure for the synthesis of indolyl-esters. In this case, a slight excess of (E)-4-bromo-2-methyl crotonaldehyde $^{3}(1.55 \mathrm{mmol}, 253 \mathrm{mg})$ respect to 1.50 of indolyl-amine was used and all the reaction was carried out at $0^{\circ} \mathrm{C}$. After completation of the reaction judged by ${ }^{1} \mathrm{H}-\mathrm{NMR}(32 \mathrm{~h})$, the reaction was quenched with $\mathrm{H}_{2} \mathrm{O}$ and the volatiles were removed under reduced pressure using a cooled rotavapor bath. The remaining aqueous phase was extracted with $\mathrm{CH}_{2} \mathrm{Cl}_{2}$ and washed with brine. After evaporation, the crude compound (viscous brown oil) was stirred in $5 \mathrm{~mL}$ of $c$-Hex until a red solid was obtained and isolated by filtration. The crude aldehyde $(\approx 10 \%$ of starting indole-amine was still detected by ${ }^{1} \mathrm{H}-\mathrm{NMR}$ ) was directly employed in the next step without further purifications. ${ }^{1} \mathrm{H}-\mathrm{NMR}\left(300 \mathrm{MHz}, \mathrm{CDCl}_{3}\right): \delta 1.68(\mathrm{~d}, J=1.2 \mathrm{~Hz}, 3 \mathrm{H}), 3.40(\mathrm{~d}, J=6.0 \mathrm{~Hz}, 2 \mathrm{H})$, $3.69(\mathrm{~s}, 2 \mathrm{H}), 3.80(\mathrm{~s}, 2 \mathrm{H}), 6.40(\mathrm{~s}, 1 \mathrm{H}), 6.58(\mathrm{dt}, J=1.5,6.3 \mathrm{~Hz}, 1 \mathrm{H}), 7.06-7.15(\mathrm{~m}, 1 \mathrm{H}), 7.17-7.21$ (m, 1H), 7.30-7.39 (m, 5H), 7.42-7.48 (m, 1H), 7.55 (d, J=7.2 Hz, 1H), 8.41 (br, 1H), 9.39 (s, 1H). LC-ESI-MS: $319(\mathrm{M}+1)$. The crude aldehyde $(1 \mathrm{mmol})$ was dissolved in anhydrous toluene (10 $\mathrm{mL}$ ) and the solution was cooled to $-78^{\circ} \mathrm{C}$. After $5 \mathrm{~min}$. stirring at this temperature, a solution of DIBAL in $n$-Hex $(1.1 \mathrm{mmol}, 1 \mathrm{M})$ was added dropwise. The reaction mixture was stirred at $-78^{\circ} \mathrm{C}$ for $2.5 \mathrm{~h}$, then $5 \mathrm{~mL}$ of $\mathrm{H}_{2} \mathrm{O}$ were added and allowing the temperature to worm up to rt. Then, the two phases were separated and the aqueous extracted with $\mathrm{CH}_{2} \mathrm{Cl}_{2}(3 \times 5 \mathrm{~mL})$. The collected organic phases were washed with brine $(5 \mathrm{~mL})$ and dried under $\mathrm{Na}_{2} \mathrm{SO}_{4}$. After removal of the volatiles, the crude was purified by flash chromatography (c-Hex:AcOEt 60:40) to leave the desired alcohol as a pale yellow oil in 35\% yield (two steps). ${ }^{1} \mathrm{H}-\mathrm{NMR}\left(200 \mathrm{MHz}, \mathrm{CDCl}_{3}\right): \delta 1.61$ (s, $3 \mathrm{H}$ ); $3.13(\mathrm{~d}, J=5.4 \mathrm{~Hz}, 2 \mathrm{H}) ; 3.63(\mathrm{~s}, 2 \mathrm{H}) ; 3.74(\mathrm{~s}, 2 \mathrm{H}) ; 3.98(\mathrm{~s}, 2 \mathrm{H}) ; 5.77(\mathrm{t}, J=6.6 \mathrm{~Hz}, 1 \mathrm{H}) ; 6.36(\mathrm{~s}$, 1H); 7.01-7.10 (m, 2H); 7.14-7.37 (m, 6H); 7.52 (d, $J=7.2 \mathrm{~Hz}, 1 \mathrm{H}) ; 8.54$ (br, 1H). ${ }^{13} \mathrm{C}-\mathrm{NMR}(50$ 
$\left.\mathrm{MHz}, \mathrm{CDCl}_{3}\right): \delta 14.0,50.2,51.2,57.7,67.7,102.9,111.2,119.8,120.2,122.0,127.8,128.0,128.3$, 128.7(2C), 129.1, 129.6(2C), 129.7, 136.4. LC-ESI-MS: $321(\mathrm{M}+1)$.

The final synthesis of the carbonate $\mathbf{1 h}$ was performed analogously to the previously described for the synthesis of indolyl-carbonates 1. (c-Hex:AcOEt 90:10) to leave the desired carbonate as a pale yellow solid in $38 \%$ yield. ${ }^{1} \mathrm{H}-\mathrm{NMR}\left(200 \mathrm{MHz}, \mathrm{CDCl}_{3}\right): \delta 1.60(\mathrm{~s}, 3 \mathrm{H}), 3.62(\mathrm{~s}, 2 \mathrm{H}), 3.73(\mathrm{~s}, 2 \mathrm{H})$, $3.79(\mathrm{~s}, 3 \mathrm{H}), 4.54(\mathrm{~s}, 2 \mathrm{H}), 5.70(\mathrm{t}, J=6.0 \mathrm{~Hz}, 1 \mathrm{H}), 3.37$ (s, 1H), 7.07-7.25 (m, 2H), 7.29-7.35 (m, $6 \mathrm{H}), 7.54$ (d, $J=9.6 \mathrm{~Hz}, 1 \mathrm{H}), 8.62$ (br, 1H). IR (neat): v 3409, 3050, 3022, 2815, 1749, 1450, 1281 $\mathrm{cm}^{-1} .{ }^{13} \mathrm{C}-\mathrm{NMR}\left(75 \mathrm{MHz}, \mathrm{CDCl}_{3}\right): \delta 14.2,50.4,51.0,54.8,58.0,72.9,101.7,110.8,119.6,120.1$, 121.2, 121.6, 126.0, 127.3, 128.4(2C), 128.7, 129.1(2C), 130.6, 136.1, 155.7, LC-ESI-MS: 379 $(\mathrm{M}+1), 401(\mathrm{M}+\mathrm{Na})$. Anal. calcd for $\left(\mathrm{C}_{23} \mathrm{H}_{26} \mathrm{~N}_{2} \mathrm{O}_{3}\right)$ : C, 72.99; H, 6.92; N, 7.40; Found: C, 72.96; H, $6.86 ; \mathrm{N}, 7.42$.

\section{Preparation of 4-vinyl-THBCs (2) and 1-vinyl-THGCs (7).}

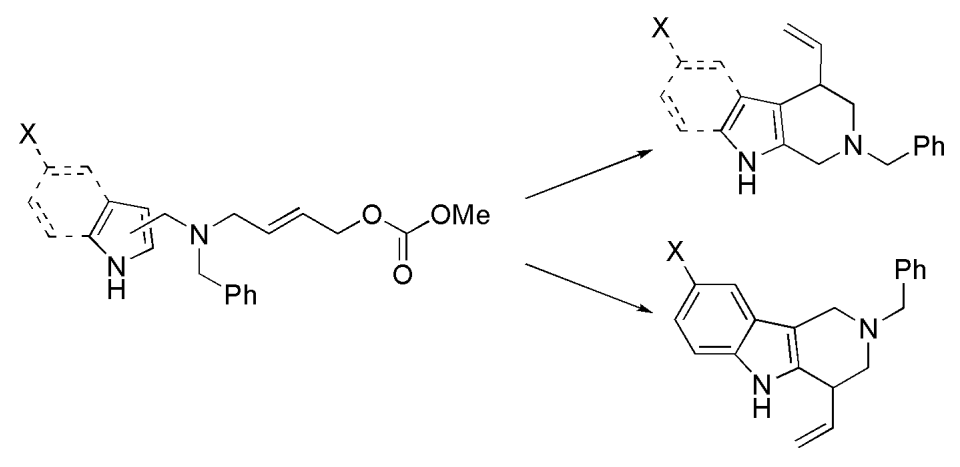

The reaction was carried out at $\mathrm{rt}$ under nitrogen atmosphere. A solution of $\left[\mathrm{Pd}_{2} \mathrm{dba}_{3}\right] \cdot \mathrm{CHCl}_{3}(3.6$ mmol, $5 \mathrm{~mol} \%)$ and the desired chiral ligand (11 mol\%) in anhydrous $\mathrm{CH}_{2} \mathrm{Cl}_{2}(1.0 \mathrm{~mL})$ was stirred until the solution colour turned from deep red to orange (about $30 \mathrm{~min}$ ). Then, the carbonate $\mathbf{1} / \mathbf{6}$ ( 0.07 mmol, 1 equiv) dissolved in $0.5 \mathrm{~mL}$ of $\mathrm{CH}_{2} \mathrm{Cl}_{2}$ and $\mathrm{Li}_{2} \mathrm{CO}_{3}$ (2 equiv) were added. The resulting reaction mixture slowly turned form yellow. The reaction was stirred overnight (complete consumption of $\mathbf{1} / \mathbf{6}$ as judged by TLC) and then quenched with water ( $4 \mathrm{~mL}$ ) and extracted with AcOEt. The combined organics were dried over $\mathrm{Na}_{2} \mathrm{SO}_{4}$ and concentrated under reduced pressure. The crude was purified by passage through a pad of silica gel and the enantiomeric excess determined through HPLC analysis with chiral column. The $C / N$ regiochemistry of the cyclization was determined on the reaction crude by HPLC and confirmed after flash chromatography.

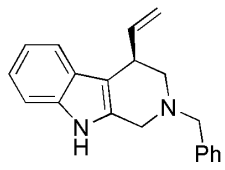

(R)-2a. Pale yellow oil. $\left(\mathrm{CH}_{2} \mathrm{Cl}_{2}\right.$ :AcOEt 8:2). Yield 95\% $(\mathrm{C} / \mathrm{N}>50: 1)$. Ee $=92 \%$. $[\alpha]^{\mathrm{D}}=+55.6$ (c $0.4, \mathrm{CHCl}_{3}$ ). HPLC analysis: OD column $(225 \mathrm{~m})$, method: $n$ Hex:IPA 90:10, flow $0.5 \mathrm{~mL} / \mathrm{min}, \mathrm{t}_{\mathrm{S}}=28.7 \mathrm{~min}, \mathrm{t}_{\boldsymbol{R}}=30.2 \mathrm{~min}$. ${ }^{1}$ 


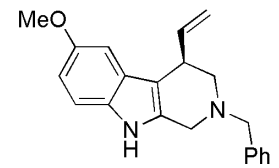

(R)-2c. Yellow solid. $\left(\mathrm{CH}_{2} \mathrm{Cl}_{2}\right.$ :AcOEt 8:2). $\mathrm{Mp}=138-141^{\circ} \mathrm{C}$. Yield 98\% $(\mathrm{C} / \mathrm{N}>$ 50:1). Ee $=90 \%,\left(\right.$ yield $=50 \%$, ee $=94 \%$ at $\left.0^{\circ} \mathrm{C}\right) .[\alpha]^{\mathrm{D}}=45.2\left(\right.$ c $\left.0.9, \mathrm{CHCl}_{3}\right)$.

HPLC analysis: AD column $(225 \mathrm{~nm})$, method: $n$-Hex:IPA 90:10 flow 0.6 $\mathrm{mL} / \mathrm{min}, \mathrm{t}_{\mathrm{S}}=19.9 \mathrm{~min}, \mathrm{t}_{R}=27.5 \mathrm{~min} .{ }^{1} \mathrm{H}-\mathrm{NMR}\left(200 \mathrm{MHz}, \mathrm{CDCl}_{3}\right): \delta 2.66(\mathrm{dd}, J=7.0,11.8 \mathrm{~Hz}$, 1H), 2.97 (dd, $J=2.6,11.8 \mathrm{~Hz}, 1 \mathrm{H}), 3.63$ (s, 3H), 3.77 (d, $J=3.0 \mathrm{~Hz}, 2 \mathrm{H}), 3.83$ (s, 3H), 5.14-5.31 (m, 2H), 5.85-6.08 (m, 1H), 6.77 (dd, $J=2.2,8.8 \mathrm{~Hz}, 1 \mathrm{H}), 7.01$ (d, $J=1.8 \mathrm{~Hz}, 1 \mathrm{H}), 7.17$ (d, $J=8.8$ $\mathrm{Hz}, 1 \mathrm{H}), 7.27-7.38(\mathrm{~m}, 5 \mathrm{H}), 7.60(\mathrm{br}, 1 \mathrm{H}) ;{ }^{13} \mathrm{C}-\mathrm{NMR}\left(75 \mathrm{MHz}, \mathrm{CDCl}_{3}\right): \delta 38.8,50.5,56.1,57.5$, 62.2, 101.9, 110.0, 110.9, 111.5, 115.7, 127.5, 127.8(2C), 128.6(2C), 129.3, 131.4, 133.3, 138.6, 140.5, 154.0; LC-ESI-MS: 319 (M+1); IR (nujol): v 3395(w), 3188(m), 2726(s), 1731(m), 1460(s), $1373(\mathrm{~s}) \mathrm{cm}^{-1}$. Anal. calcd for $\left(\mathrm{C}_{21} \mathrm{H}_{22} \mathrm{~N}_{2} \mathrm{O}\right): \mathrm{C}, 79.21 ; \mathrm{H}, 6.69 ; \mathrm{N}, 8.80$; Found: C, 79.05; H, 6.60; N, 8.77 .

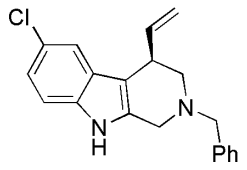

(R)-2d. Yellow solid. $\left(\mathrm{CH}_{2} \mathrm{Cl}_{2}\right.$ :AcOEt 95:5). $\mathrm{Mp}=140-142^{\circ} \mathrm{C}$. Yield $82 \%(\mathrm{C} / \mathrm{N}>$ 50:1). Ee $=82 \% .[\alpha]^{\mathrm{D}}=+48.7\left(c \quad 0.2, \mathrm{CHCl}_{3}\right)$. HPLC analysis: OD column (225/280 nm), method: $n$-Hex:IPA 93:7 flow $0.5 \mathrm{~mL} / \mathrm{min}, \mathrm{t}_{R}=39.6 \mathrm{~min}, \mathrm{t}_{\mathrm{S}}=45.2$ min. ${ }^{1} \mathrm{H}-\mathrm{NMR}\left(200 \mathrm{MHz}, \mathrm{CDCl}_{3}\right): \delta 2.67(\mathrm{dd}, J=7.0,11.8 \mathrm{~Hz}, 1 \mathrm{H}), 3.02(\mathrm{dd}, J=5.2,11.8 \mathrm{~Hz}$, $1 \mathrm{H}), 3.64(\mathrm{~s}, 2 \mathrm{H}), 3.66-3.69(\mathrm{~m}, 1 \mathrm{H}), 3.77$ (d, $J=2.6 \mathrm{~Hz}, 2 \mathrm{H}), 5.21(\mathrm{t}, J=8.4 \mathrm{~Hz}, 1 \mathrm{H}), 5.33(\mathrm{~s}, 1 \mathrm{H})$, 5.83-6.01 (m, 1H), $7.10(\mathrm{dd}, J=2.2,8.8 \mathrm{~Hz}, 1 \mathrm{H}), 7.19-7.51(\mathrm{~m}, 7 \mathrm{H}), 7.85$ (br, $1 \mathrm{H}) ;{ }^{13} \mathrm{C}-\mathrm{NMR}(50$ $\left.\mathrm{MHz}, \mathrm{CDCl}_{3}\right): \delta 30.9,37.6,49.7,60.4,109.0,111.9,116.9,118.5,121.8,125.1,127.6(2 \mathrm{C})$, 128.1(2C), 128.6, 129.6, 134.6, 136.6, 138.8, 155.1. ESI-MS: 322 (M+1); IR (neat): v 3399(s), 2999(m), 2950(s), 2826(s), 1749(s), 1528(m), 1490(w), 1257(s) $\mathrm{cm}^{-1}$. Anal. calcd for $\left(\mathrm{C}_{20} \mathrm{H}_{19} \mathrm{ClN}_{2}\right)$ : C, 74.41; H, 5.93; N, 8.68; Found: C, 74.37; H, 5.89; N, 8.65.

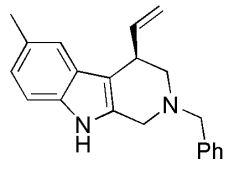

(R)-2e. Pale yellow solid. $\left(\mathrm{CH}_{2} \mathrm{Cl}_{2}\right.$ :AcOEt $\left.95: 5\right) . \mathrm{Mp}=152-153^{\circ} \mathrm{C}$. Yield $95 \%$ $(C / N>50: 1) . \mathrm{Ee}=97 \% .[\alpha]^{\mathrm{D}}=+63.0\left(c 0.3, \mathrm{CHCl}_{3}\right)$. HPLC analysis: OD column (225 nm), method: $n$-Hex:IPA 92:8 flow $0.5 \mathrm{~mL} / \mathrm{min}, \mathrm{t}_{S}=30.8 \mathrm{~min}, \mathrm{t}_{R}=32.1$ min. ${ }^{1} \mathrm{H}-\mathrm{NMR}\left(300 \mathrm{MHz}, \mathrm{CDCl}_{3}\right): \delta 2.42(\mathrm{~s}, 3 \mathrm{H}), 2.67(\mathrm{dd}, J=6.9,11.4,1 \mathrm{H}), 2.97$ (dd, $J=4.8$, $11.4 \mathrm{~Hz}, 1 \mathrm{H}), 3.63$ (d, $J=11.7 \mathrm{~Hz}, 2 \mathrm{H}), 3.70-3.74(\mathrm{~m}, 1 \mathrm{H}), 3.77$ (d, $J=11.7 \mathrm{~Hz}, 2 \mathrm{H}), 5.16$ (dd, $J=$ 1.5, $9.9 \mathrm{~Hz}, 1 \mathrm{H}), 5.30(\mathrm{~d}, J=16.2 \mathrm{~Hz}, 1 \mathrm{H}), 5.92-6.03(\mathrm{~m}, 1 \mathrm{H}), 6.94(\mathrm{~d}, J=8.1 \mathrm{~Hz}, 1 \mathrm{H}), 7.16(\mathrm{~d}, J=$ $9.0 \mathrm{~Hz}, 1 \mathrm{H}), 7.29-7.41(\mathrm{~m}, 6 \mathrm{H}), 7.59(\mathrm{br}, 1 \mathrm{H}) .{ }^{13} \mathrm{C}-\mathrm{NMR}\left(75 \mathrm{MHz}, \mathrm{CDCl}_{3}\right): \delta 21.5,38.4,50.2,57.3$, 61.9, 109.5, 110.3, 115.3, 118.8, 122.7, 127.2, 127.3(2C), 128.3(2C), 128.5, 129.0, 132.2, 134.4, 138.4, 140.5. ESI-MS: 303 (M+1); IR (nujol): v 3386(s), 2925(s), 2854(s), 1450(s), 1346(m) cm ${ }^{-1}$. Anal. calcd for $\left(\mathrm{C}_{21} \mathrm{H}_{22} \mathrm{~N}_{2}\right)$ : C, 83.40; H, 7.33; N, 9.26; Found: C, 83.35; H, 7.27; N, 9.25. 


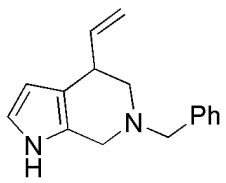

2f. Viscous brown oil. (c-Hex:AcOEt 90:10). Yield 85\% $(C / N>50: 1)$. Ee $=95 \%$. $[\alpha]^{\mathrm{D}}=-23.6\left(\right.$ c $\left.0.36, \mathrm{CHCl}_{3}\right)$. HPLC analysis: OD column $(214 \mathrm{~nm})$, method: $n$ Hex:IPA 90:10 flow $0.5 \mathrm{~mL} / \mathrm{min}, \mathrm{t}-(+)=12.3 \mathrm{~min}, \mathrm{t}-(-)=13.2 \mathrm{~min} .{ }^{1} \mathrm{H}-\mathrm{NMR}(200$ $\left.\mathrm{MHz}, \mathrm{CDCl}_{3}\right): \delta 2.47(\mathrm{dd}, J=8.4,12.4 \mathrm{~Hz}, 1 \mathrm{H}), 3.01(\mathrm{dd}, J=6.0 \mathrm{~Hz}, 1 \mathrm{H}), 3.42-3.62(\mathrm{~m}, 2 \mathrm{H}), 5.13$ $(\mathrm{dd}, J=1,4,9.8 \mathrm{~Hz}, 1 \mathrm{H}), 5.18-5.27(\mathrm{~m}, 1 \mathrm{H}), 5.71-5.88(\mathrm{~m}, 1 \mathrm{H}), 5.96(\mathrm{t}, J=2,6 \mathrm{~Hz}, 1 \mathrm{H}), 6.56(\mathrm{t}, J$ $=3.0 \mathrm{~Hz}, 1 \mathrm{H}), 7.30-7.41(\mathrm{~m}, 5 \mathrm{H}), 7.82(\mathrm{br}, 1 \mathrm{H}) .{ }^{13} \mathrm{C}-\mathrm{NMR}\left(50 \mathrm{MHz}, \mathrm{CDCl}_{3}\right): \delta 39.6,51.1,56.3$, 62.0, 105.7, 117.1, 119.2, 121.2, 127.2, 127.4, 128.4(2C), 129.3(2C), 132.0, 138.4. ESI-MS: 239 $(\mathrm{M}+1)$; IR (nujol): $v$ 3392(s), 2932(s), 2860(s), 1459(s), $1357(\mathrm{~m}) \mathrm{cm}^{-1}$. Anal. calcd for $\left(\mathrm{C}_{16} \mathrm{H}_{18} \mathrm{~N}_{2}\right)$ : C, 80.63; H, 7.61; N, 11.75; Found: C, 80.60; H, 7.58; N, 11.72.

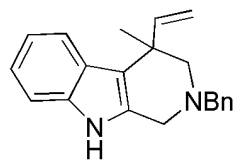

2g. Viscous brown oil. (c-Hex:AcOEt 90:10). Yield 45\% (C/N> 50:1). Ee = 90\%. $[\alpha]^{\mathrm{D}}=-15.2\left(\right.$ c $\left.0.5, \mathrm{CHCl}_{3}\right)$. HPLC analysis: OD column $(214 \mathrm{~nm})$, method: $n$ Hex:IPA 97:3 flow $0.5 \mathrm{~mL} / \mathrm{min}, \mathrm{t}-(-)=38.9 \mathrm{~min}, \mathrm{t}-(+)=41.9 \mathrm{~min} .{ }^{1} \mathrm{H}-\mathrm{NMR}(200$ $\left.\mathrm{MHz}, \mathrm{CDCl}_{3}\right): \delta 1.60(\mathrm{~s}, 3 \mathrm{H}), 2.55(\mathrm{~d}, J=11.6 \mathrm{~Hz}, 1 \mathrm{H}), 2.68(\mathrm{~d}, J=11.6 \mathrm{~Hz}, 1 \mathrm{H}), 3.64(\mathrm{~s}, 2 \mathrm{H})$, $3.77(\mathrm{~s}, 2 \mathrm{H}), 5.01(\mathrm{~s}, 1 \mathrm{H}), 5.14(\mathrm{~d}, J=6.0 \mathrm{~Hz}, 1 \mathrm{H}), 6.09$ (dd, $J=10.6,16.4 \mathrm{~Hz}, 1 \mathrm{H}), 7.03-7.16(\mathrm{~m}$, 2H), 7.35-7.46 (m, 6H), $7.56(\mathrm{~d}, J=5.2 \mathrm{~Hz}, 1 \mathrm{H}), 7.69(\mathrm{br}, 1 \mathrm{H}) .{ }^{13} \mathrm{C}-\mathrm{NMR}\left(50 \mathrm{MHz}, \mathrm{CDCl}_{3}\right): \delta$ 26.9, 39.0, 51.0, 62.5, 64.1, 110.8, 112.6, 119.1, 119.8, 121.1, 121.7, 125.7, 128.3(2C), 128.8(2C), 132.2, 133.9, 145.2 ESI-MS: 303 (M+1); IR (nujol): v 3380(s), 2926(s), 2856(s), 1451(s), 1351(m) $\mathrm{cm}^{-1}$. Anal. calcd for $\left(\mathrm{C}_{21} \mathrm{H}_{22} \mathrm{~N}_{2}\right)$ : C, 83.40; H, 7.33; N, 9.26; Found: C, 83.35; H, 7.30; N, 9.22.

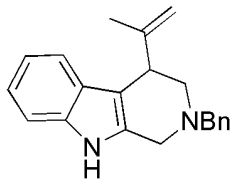

2h. Viscous brown oil. (c-Hex:AcOEt 90:10). Yield 49\% $(C / N>50: 1)$. Ee $=94 \%$. $[\alpha]^{\mathrm{D}}=-18.9\left(\right.$ c $\left.0.1, \mathrm{CHCl}_{3}\right)$. HPLC analysis: OD column $(214 \mathrm{~nm})$, method: $n$ Hex:IPA 87:13 flow $0.5 \mathrm{~mL} / \mathrm{min}, \mathrm{t}-(-)=18.6 \mathrm{~min}, \mathrm{t}-(+)=21.4 \mathrm{~min} .{ }^{1} \mathrm{H}-\mathrm{NMR}(200$ $\left.\mathrm{MHz}, \mathrm{CDCl}_{3}\right): \delta 1.64(\mathrm{~s}, 3 \mathrm{H}), 2.72(\mathrm{dd}, J=3.6,10.4 \mathrm{~Hz}, 1 \mathrm{H}), 3.03(\mathrm{dd}, J=5.8,10.4,1 \mathrm{H}), 3.11(\mathrm{~d}, J$ $=6.2 \mathrm{~Hz}, 1 \mathrm{H}), 3.66(\mathrm{~s}, 2 \mathrm{H}), 3.85(\mathrm{~s}, 2 \mathrm{H}), 4.96(\mathrm{~d}, J=5.6 \mathrm{~Hz}, 2 \mathrm{H}), 7.02-7.16(\mathrm{~m}, 2 \mathrm{H}), 7.32-7.45(\mathrm{~m}$, $6 \mathrm{H}), 7.55(\mathrm{~d}, J=7.2 \mathrm{~Hz}, 1 \mathrm{H}), 8.44(\mathrm{br}, 1 \mathrm{H}) .{ }^{13} \mathrm{C}-\mathrm{NMR}\left(50 \mathrm{MHz}, \mathrm{CDCl}_{3}\right): \delta$ 19.9, 41.8, 50.1, 56.7, $61.9,109.9,110.6,112.8,119.1,119.3,120.0,121.2,127.2,128.3(2 \mathrm{C}), 129.0(2 \mathrm{C}), 132.7,136.0$, 138.4, 146.6. ESI-MS: 303 (M+1); IR (nujol): v 3375(s), 2930(s), 2875(s), 1450(s), 1328(m) $\mathrm{cm}^{-1}$. Anal. calcd for $\left(\mathrm{C}_{21} \mathrm{H}_{22} \mathrm{~N}_{2}\right)$ : C, 83.40; H, 7.33; N, 9.26; Found: C, 83.35; H, 7.29; N, 9.20.

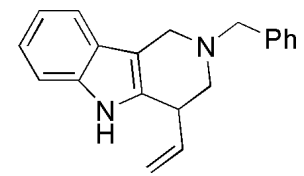

7a. Yellow solid. (c-Hex:AcOEt 7:3). Yield 93\% $(C / N>50: 1)$. Ee $=93 \%$. $[\alpha]^{\mathrm{D}}=+22.5\left(\right.$ c $\left.0.7, \mathrm{CHCl}_{3}\right) . \mathrm{Mp}=109-113^{\circ} \mathrm{C} . \mathrm{HPLC}$ analysis: OD column $(225$ 
nm), method: $n$-Hex:IPA 90:10 flow $0.5 \mathrm{~mL} / \mathrm{min}, \mathrm{t}-(+)=26.5 \mathrm{~min}, \mathrm{t}-(-)=27.9 \mathrm{~min} .{ }^{1} \mathrm{H}-\mathrm{NMR}(300$ $\left.\mathrm{MHz}, \mathrm{CDCl}_{3}\right): \delta 2.70(\mathrm{dd}, J=6.6,11.4 \mathrm{~Hz}, 1 \mathrm{H}), 3.03(\mathrm{dd}, J=5.2,11.4 \mathrm{~Hz}, 1 \mathrm{H}), 3.69-3.71(\mathrm{~m}, 2 \mathrm{H})$, $3.72-3.74(\mathrm{~m}, 1 \mathrm{H}), 3.80(\mathrm{~d}, J=2.8 \mathrm{~Hz}, 2 \mathrm{H}), 5.17(\mathrm{dd}, J=1.5,9.9,1 \mathrm{H}), 5.29(\mathrm{dd}, J=1.5,17.1 \mathrm{~Hz}$, 1H), 5.91-6.03 (m, 1H), 7.05-7.16 (m, 2H), 7.29-7.43 (m, 6H), $7.55(\mathrm{~d}, J=7.0,1 \mathrm{H}), 7.72(\mathrm{br}, 1 \mathrm{H})$; ${ }^{13} \mathrm{C}-\mathrm{NMR}\left(75 \mathrm{MHz}, \mathrm{CDCl}_{3}\right): \delta 38.7,50.2,57.3,62.0,110.1,111.0,115.9,119.4,119.6,121.6$, 117.6, 128.2(2C), 128.7(2C), 129.4, 132.0, 136.3, 138.1, 140.4; LC-ESI-MS: 289 (M+1); IR (neat): v 3399(m), 3256(s), 3058(s), 2920(s), 2810(m), 1615(w), 1494(m), 1455(s), 1257(s), 1098(m) cm ${ }^{-1}$. Anal. calcd for $\left(\mathrm{C}_{20} \mathrm{H}_{20} \mathrm{~N}_{2}\right)$ : C, 83.30; H, 6.99; N, 9.71; Found: C, 83.22; H, 6.94; N, 9.70.

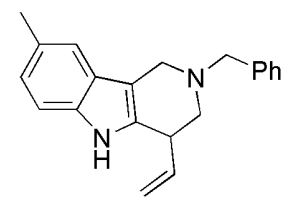

7b. Yellow solid. (c-Hex:AcOEt 7:3). Yield 82\% $(C / N>50: 1) . \mathrm{Ee}=92 \% .[\alpha]^{\mathrm{D}}=$ $+36.2\left(\right.$ c $\left.0.3, \mathrm{CHCl}_{3}\right) . \mathrm{Mp}=134-136^{\circ} \mathrm{C}$. HPLC analysis: OD column $(225 \mathrm{~nm})$, method: $n$-Hex:IPA 92:8 flow $0.5 \mathrm{~mL} / \mathrm{min}, \mathrm{t}-(-)=31.3 \mathrm{~min}, \mathrm{t}-(+)=32.8 \mathrm{~min}$. ${ }^{1} \mathrm{H}-\mathrm{NMR}\left(200 \mathrm{MHz}, \mathrm{CDCl}_{3}\right): \delta 2.37(\mathrm{~s}, 3 \mathrm{H}), 2.65(\mathrm{dd}, J=2.8,6.8 \mathrm{~Hz}, 1 \mathrm{H}), 2.91-3.00(\mathrm{~m}, 1 \mathrm{H})$, 3.58-3.62 (m, 2H), 3.65-3.68 (m, 1H), 3.70-3.72 (m, 2H), 5.08 (d, $J=9.8 \mathrm{~Hz}, 1 \mathrm{H}), 5.21(\mathrm{~d}, J=16.6$ $\mathrm{Hz}, 1 \mathrm{H}), 5.82-6.08(\mathrm{~m}, 1 \mathrm{H}), 6.86(\mathrm{~d}, J=8.0 \mathrm{~Hz}, 1 \mathrm{H}), 7.11-7.18(\mathrm{~m}, 1 \mathrm{H}), 7.21-7.34(\mathrm{~m}, 6 \mathrm{H}), 7.57$ (br, $1 \mathrm{H}) ;{ }^{13} \mathrm{C}-\mathrm{NMR}\left(50 \mathrm{MHz}, \mathrm{CDCl}_{3}\right): \delta 21.5,38.4,50.2,57.3,61.9,110.3,115.3,118.8,122.7$, 127.2, 128.0(2C), 128.3(2C), 129.0, 129.4, 132.5, 134.4, 136.4, 138.8, 140.6; LC-ESI-MS: 303 $(\mathrm{M}+1)$; IR (neat): v 3396(s), 2962(m), 2907(m), 1653(s), 1451(w), 1263(m), 1073(m) $\mathrm{cm}^{-1}$. Anal. calcd for $\left(\mathrm{C}_{21} \mathrm{H}_{22} \mathrm{~N}_{2}\right)$ : C, 83.40; H, 7.33; N, 9.26; Found: C, 83.35; H, 7.29; N, 9.25.

\section{Preparation of the $(Z)-3$-indolyl-methyl carbonate.}

To a cooled solution $\left(0^{\circ} \mathrm{C}\right)$ of 3-indolyl-amine $(0.3 \mathrm{mmol}, 70 \mathrm{mg})$ and $\mathrm{Et}_{3} \mathrm{~N}(50 \mu \mathrm{L}, 0.6 \mathrm{mmol})$ in dry $\mathrm{CH}_{2} \mathrm{Cl}_{2}$ (5 mL), (Z)-(4-bromobut-2-en-1-yl) methyl carbonate ${ }^{4}$ ( $85 \mathrm{mg}, 0.33 \mathrm{mmol}$ ) dissolved in $3 \mathrm{~mL}$ of $\mathrm{CH}_{2} \mathrm{Cl}_{2}$ was added. The ice bath was removed and the reaction mixture was stirred at $\mathrm{rt}$ overnight. Then the reaction was quenched with water $(5 \mathrm{~mL})$ and the organic phases washed with $\mathrm{HCl}(1 \mathrm{~N}, 5 \mathrm{~mL})$. After evaporation of the volatiles under reduced pressure the desired carbonate was purified by flash chromatography with $c$-Hex:AcOEt 8:2 as the eluent mixture.

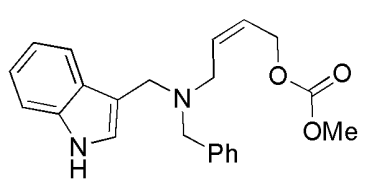

Viscous yellow oil. Yield 81\% (88 mg). ${ }^{1} \mathrm{H}-\mathrm{NMR}\left(200 \mathrm{MHz}, \mathrm{CDCl}_{3}\right): \delta$

$3.15(\mathrm{~d}, J=6.6 \mathrm{~Hz}, 2 \mathrm{H}), 3.62(\mathrm{~s}, 2 \mathrm{H}), 3.78(\mathrm{~s}, 5 \mathrm{H}), 4.63(\mathrm{~d}, J=6.6 \mathrm{~Hz}$,

2H), 5.64-5.78 (m, 1H), 5.84-5.95 (m, 1H), 7.16-7.24 (m, 3H), 7.31-7.42

$(\mathrm{m}, 6 \mathrm{H}), 7.74(\mathrm{~d}, J=6.8 \mathrm{~Hz}, 1 \mathrm{H}), 8.03(\mathrm{br}, 1 \mathrm{H}) ;{ }^{13} \mathrm{C}-\mathrm{NMR}\left(75 \mathrm{MHz}, \mathrm{CDCl}_{3}\right): \delta 50.0,50.9,54.9$, 58.2 , 63.4, 101.7, 110.8, 119.5, 120.0, 121.4, 126.5, 127.2, 128.4(2C), 129.0(2C), 132.3, 136.1, 138.4, 155.8; LC-ESI-MS: 365 (M+1); IR (nujol): v 3414(s), 2960(m), 2924 (s), 1750(s), 1456 (m), 
1375(w), 1264(m), 739(w), 418(s) $\mathrm{cm}^{-1}$. Anal. calcd for $\left(\mathrm{C}_{22} \mathrm{H}_{24} \mathrm{~N}_{2} \mathrm{O}_{3}\right): \mathrm{C}, 72.50 ; \mathrm{H}, 6.64 ; \mathrm{N}, 7.69$;

Found: C, 72.39; H, 6.62; N, 7.63 .

\section{Determination of the absolute configuration.}

\section{1) X-Ray analysis}

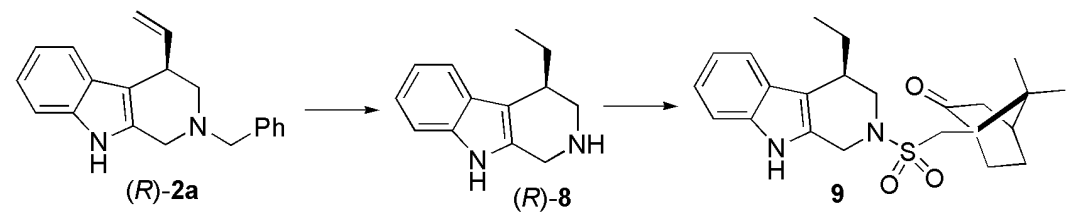

Cleavage of the benzyl group. The tetrahydro- $\beta$-carboline $(S)-2 a$ (ee: $65 \%, 60 \mathrm{mg}, 0.21 \mathrm{mmol}$ ) was added to a suspension of $\mathrm{Pd}(\mathrm{OH})_{2}(20 \mathrm{~mol} \%)$ in anhydrous $\mathrm{MeOH}(4 \mathrm{~mL})$. The apparatus was connected to a balloon filled with hydrogen in order to maintain a reducing atmosphere $(\approx 1 \mathrm{~atm})$. The dark mixture was stirred at room temperature overnight. Then the insoluble black powder was filtered off (CAUTION!) with celite, washed with $\mathrm{Et}_{2} \mathrm{O}(5 \mathrm{~mL})$ and the organics evaporated under reduced pressure. The crude 8 was finally purified by flash chromatography $\left(\mathrm{CH}_{2} \mathrm{Cl}_{2}: \mathrm{MeOH} 92: 8\right)$ to live a brown solid. Yield $=95 \%(40 \mathrm{mg})$, ee $=65 \% .[\alpha]^{\mathrm{D}}=+45.7\left(\right.$ c $\left.0.7, \mathrm{CHCl}_{3}\right) . \mathrm{Mp}=119$ $121^{\circ} \mathrm{C}$. HPLC analysis: OD column $(225 \mathrm{~nm})$, method: $n$-Hex:IPA 85:15 flow $0.5 \mathrm{~mL} / \mathrm{min}, \mathrm{t}_{S}=$ $33.3 \mathrm{~min}, \mathrm{t}_{R}=35.2 \mathrm{~min} .{ }^{1} \mathrm{H}-\mathrm{NMR}\left(200 \mathrm{MHz}, \mathrm{CDCl}_{3}\right): \delta 1.03(\mathrm{t}, J=7.4 \mathrm{~Hz}, 3 \mathrm{H}), 1.58-1.69(\mathrm{~m}, 2 \mathrm{H})$, 1.97-2.05 (m, 2H), 3.07 (dq, $J=4.8,14.2 \mathrm{~Hz}, 2 \mathrm{H}), 4.00(\mathrm{~s}, 2 \mathrm{H}), 7.09-7.15(\mathrm{~m}, 2 \mathrm{H}), 7.27-7.32(\mathrm{~m}$, 1H), 7.53-7.60 (m, 1H), 8.05 (br, $1 \mathrm{H}) ;{ }^{13} \mathrm{C}-\mathrm{NMR}\left(75 \mathrm{MHz}, \mathrm{CDCl}_{3}\right): \delta 11.8,26.3,34.2,42.7,47.5$, 110.9, 112.2, 118.7, 119.2, 121.2, 127.0, 131.8, 135.8; LC-ESI-MS: $201(\mathrm{M}+1)$.

\section{Synthesis of the THBC-camphorsulfonamide derivative (9).}

To a solution of enantiomerically enriched $(R)-4$-ethyl-THBC $(10 \mathrm{mg}, 0.05 \mathrm{mmol}$, ee $=65 \%)$ in 2 $\mathrm{mL}$ of dry $\mathrm{CH}_{2} \mathrm{Cl}_{2}$ were added $6 \mathrm{mg}(0.056 \mathrm{mmol})$ of DMAP. The solution was cooled to $0^{\circ} \mathrm{C}$, stirred $10 \mathrm{~min}$ then $13 \mathrm{mg}(0.055 \mathrm{mmol})$ of $(1 R)$-(-)-10-camphorsulfonyl chloride were added. The reaction mixture was allowed to stir at $\mathrm{rt}$ for $10 \mathrm{~h}$ then quenched with water $(5 \mathrm{~mL})$, extracted with $\mathrm{CH}_{2} \mathrm{Cl}_{2}$ and the collected organic phases washed with diluted $\mathrm{HCl}$. After evaporation under reduced pressure, the diastereoisomeric sulfonamide mixture (9) was purified by flash chromatography by using $\mathrm{CH}_{2} \mathrm{Cl}_{2}$ : $\mathrm{Et}_{2} \mathrm{O} 7: 3$ as the eluent mixture.

9. White solid. Yield 55\% (11 mg, 82:18 diatereoisomeric mixture). LC-ESI-MS: $\operatorname{Rt}_{(R, R)}=14.6$ min, $415(\mathrm{M}+1), 437(\mathrm{M}+\mathrm{Na}), 453(\mathrm{M}+\mathrm{K})$, see HPLC spectra. $\mathrm{Rt}_{(S, R)}=14.9 \mathrm{~min}, 415(\mathrm{M}+1), 437$ $(\mathrm{M}+\mathrm{Na}), 453(\mathrm{M}+\mathrm{K})$.

Crystals of $(S, R)-9$ (minor diastereoisomer) were obtained by slow diffusion of petroleum ether into a solution of 9 in acetone. Some crystals of $(S, R)-9$ were then injected in LC-MS in order to assign the absolute $\mathrm{C}(4)$ configuration of both diastereoisomers (Figure S1). 
Crystal data for $(S, R)-\mathbf{9}$ collected on a Bruker APEX II CCD diffractometer (Mo-K $\mathrm{K}_{\alpha}$ radiation, $\lambda=$ $0.71073 \AA$ ) Empirical absorption correction was applied, initial structure model by direct methods. Anisotropic full-matrix least-squares refinement on $F^{2} \cdot \mathrm{C}_{23} \mathrm{H}_{30} \mathrm{~N}_{2} \mathrm{O}_{3} \mathrm{~S}, \mathrm{M}_{\mathrm{r}}=414.55$, Orthorhombic, $\mathrm{P} 2{ }_{1} 22_{1}, a=8.7131(15), b=10.2665(17), c=24.621(4) \AA, \mathrm{V}=2202.4(6) \AA^{3}, \mathrm{Z}=4, \mathrm{D}_{\mathrm{x}}=1.250$ $\mathrm{Mg} / \mathrm{m}^{3}, \mu=0.173 \mathrm{~mm}^{-1}, \mathrm{~F}(000)=888, \mathrm{~T}=293(2) \mathrm{K}$, theta $\max 28.66^{\circ}, 18802$ reflections collected, 3717 reflections $I>2 \sigma(\mathrm{I})$. Final agreement indices $\mathrm{R}_{1}=0.0413$ and $w \mathrm{R}_{2}=0.1051$. Goodness-of-fit $=0.98$, absolute structure parameter $=-0.09(7), \operatorname{CCDC} 275640$ contains the supplementary crystallographic data for this crystal structure.

\section{Figure S1}

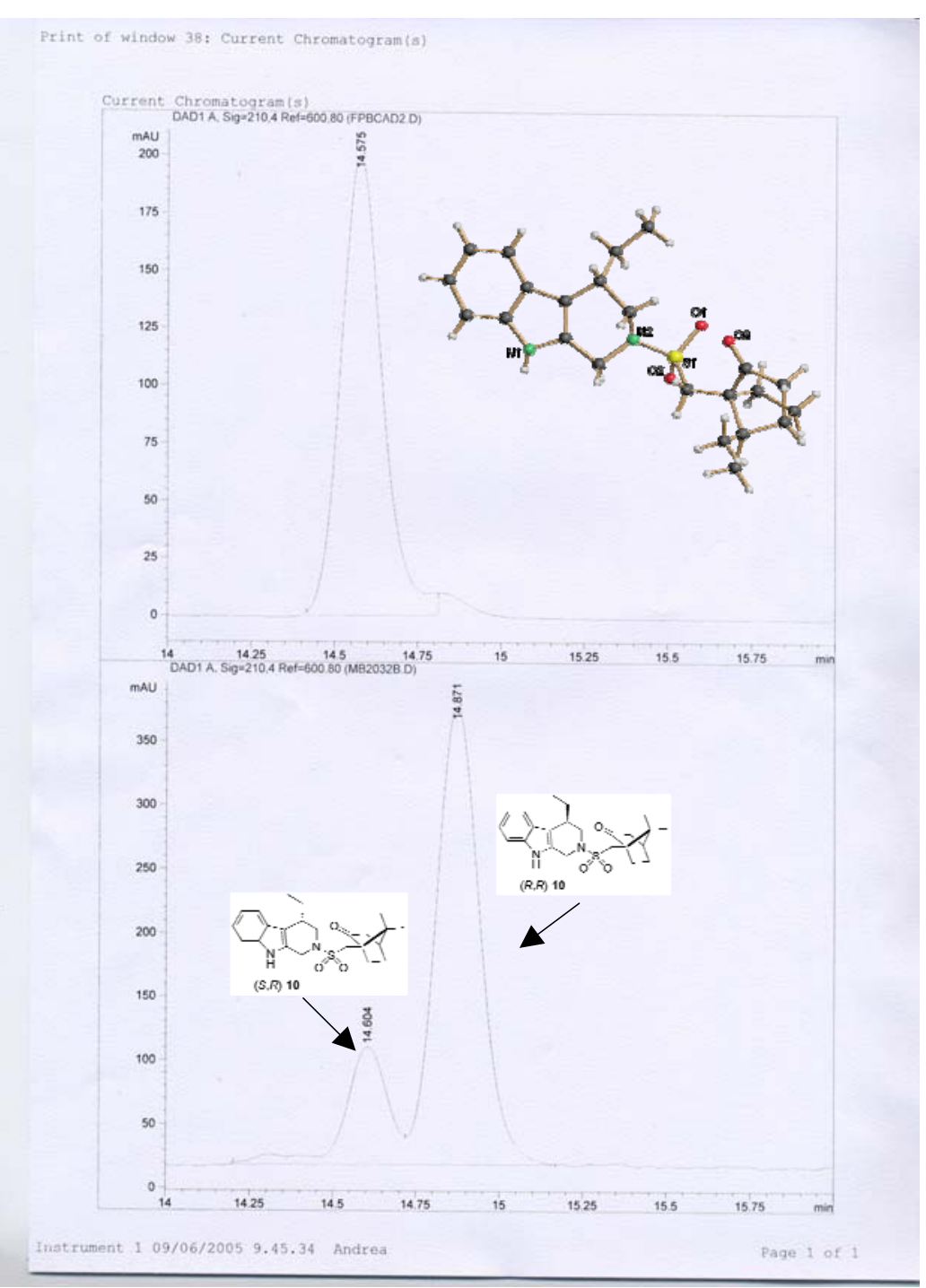




\footnotetext{
${ }^{1}$ The 2-indolylcarboxyaldehydes can be easily synthesized following a known procedure, see: Bandini, M.; Melloni, A. Umani-Ronchi, A. Org. Lett. 2004, 6, 3199. 4f, 5a,b are commercially available (Aldrich and co.)

${ }^{2}$ (E)-Ethyl 4-bromo-3-methylcrotonate was obtained by In particular, $(E)$-4-bromo-3-methyl-2-butenoyl chloride was synthesized from the corresponding carboxylic acid with $(\mathrm{COCl})_{2} / \mathrm{DMF}_{\text {cat }}$ in $\mathrm{CH}_{2} \mathrm{Cl}_{2}(\mathrm{rt}, 1.5 \mathrm{~h})$. The crude ester was obtained through a modification of the known procedure, see: Jeon, H.-S.; Yeo, J.E.; Jeong, Y.C.; Koo, S. Synthesis, 2004, 2913, (EtOLi at $\left.-78^{\circ} \mathrm{C}\right) .{ }^{1} \mathrm{H}-\mathrm{NMR}\left(200 \mathrm{MHz}, \mathrm{CDCl}_{3}\right): \delta 1.30(\mathrm{t}, J=7.4 \mathrm{~Hz}, 3 \mathrm{H}), 2.28(\mathrm{~s}, 3 \mathrm{H}), 3.95(\mathrm{~s}, 2 \mathrm{H}), 4.18$ (q, $J=7.4 \mathrm{~Hz}, 2 \mathrm{H}), 5.96(\mathrm{~s}, 1 \mathrm{H})$.

${ }^{3}$ Gray, G.M. Synlett 1983, 488.

${ }^{4}$ Oppolzer, W.; Fürstner, A. Helv. Chim. Acta 1993, 76, 2329.
} 